\title{
Homotopy classes of total foliations
}

Masayuki Asaoka, Emmanuel Dufraine and Takeo Noda*

\begin{abstract}
On every compact and orientable three-manifold we construct total foliations (three codimension-one foliations that are transverse at every point). This construction can be performed on any homotopy class of plane fields with vanishing Euler class.

As a corollary we obtain similar results on bi-contact structures.
\end{abstract}

Mathematics Subject Classification (2010). 57R30.

Keywords. Foliations, bi-contact structures.

\section{Introduction}

1.1. Main results. Let $M$ be an oriented closed three-dimensional manifold. We call a triple $\left(\xi^{i}\right)_{i=1}^{3}$ of smooth transversely oriented plane fields on $M$ a total plane field if $\bigcap_{i=1}^{3} \xi^{i}(p)=\{0\}$ for any $p$ in $M$. If each $\xi^{i}$ is integrable, it is called a total foliation. We say two total plane fields are homotopic if they are connected by a continuous path in the space of smooth oriented total plane fields.

A celebrated theorem due to Wood [16] showed that any plane field on a closed three-dimensional manifold can be continuously deformed into a foliation in its homotopy class. In other words, there is no homotopical obstruction to the integrability for the three-dimensional case. The main subject of this paper is to solve the analogous problem for total foliations. That is,

Theorem 1.1. Any total plane field on a closed three-dimensional manifold is homotopic to a total foliation.

In other words, there is no homotopical obstruction to the integrability for total plane fields.

Let us remark that three-dimensional closed manifolds have their Euler characteristic equal to zero, which implies the existence of transversely oriented plane

\footnotetext{
* The first and third author were partially supported by JSPS PostDoctoral Fellowships for Research Abroad. They also are partially supported by Grant-in-Aid for Young Scientists (B) No. 19740085 and No. 19740026 from MEXT Japan.
} 
fields. Similarly, three-dimensional closed manifolds have vanishing second StiefelWhitney class, which implies the existence of total plane fields.

Hardorp [10], showed that any three-dimensional oriented closed manifold admits a total foliation. However, his construction does not allow to keep track of the homotopy class of the constructed object.

Tamura and Sato [15], gave examples of foliations on three-dimensional manifold which admit a transverse plane field but no transverse foliation. It implies that there exists an obstruction to deform a total plane field into a total foliation if we fix one of the plane fields as a given foliation.

Mitsumatsu [13], Problem 5.2.7, asked which homotopy classes of plane fields can be realized as a transverse pair of codimension-one foliations. His question is important from the viewpoint of bi-contact structures, which we consider in the next paragraph. The theory of characteristic classes tells that a plane field is contained in a total plane field if and only if its Euler class vanishes. Theorem 1.1 answers Mitsumatsu's question immediately.

Corollary 1.2. An oriented plane field on an oriented closed three-dimensional manifold is homotopic to a foliation which is contained in a total foliation if and only if its Euler class vanishes.

We call a pair of mutually transverse positive and negative contact structures a bi-contact structure. Mitsumatsu [12], and Eliashberg and Thurston [7] showed that bi-contact structures naturally correspond to a projectively Anosov flow, which exhibits partially-hyperbolic behavior on the whole manifold.

In relation with the question above, Mitsumatsu asked which homotopy class of plane field can be realized by contact structures in a bi-contact structure. In Theorem 2.4.1 of [7], Eliashberg and Thurston showed that any foliation except the product foliation $\left\{S^{2} \times\{p\}\right\}_{p \in S^{1}}$ on $S^{2} \times S^{1}$ can be $C^{0}$-approximated by positive or negative contact structures. It is easy to see that any mutually transverse plane fields are homotopic to each other and that the product foliation on $S^{2} \times S^{1}$ does not admit a transverse foliation. Hence, the following is an immediate consequence of Eliashberg-Thurston's theorem and Corollary 1.2.

Corollary 1.3. On any oriented closed three-dimensional manifold, any oriented plane field with Euler class zero is homotopic to positive and negative contact structures which form a bi-contact structure.

Among the realization problems of bi-contact structures, the following is quite natural.

Question 1.4. Let $\xi$ and $\eta$ be positive and negative contact structures on an oriented three-dimensional manifold $M$. Suppose that they are contained in the same homo- 
topy class of plane fields with vanishing Euler class. Can we isotope $\xi$ and $\eta$ so that $(\xi, \eta)$ is a bi-contact structure?

We give an answer for overtwisted contact structures.

Theorem 1.5. Let $\xi$ and $\eta$ be positive and negative overtwisted contact structures contained in the same homotopy class of plane fields and with Euler class zero. Then, we can isotope $\xi$ and $\eta$ so that $(\xi, \eta)$ is a bi-contact structure.

The answer for tight contact structures is still unknown.

1.2. Outline of proofs. The proof of Theorem 1.1 is obtained after performing a sequence of surgeries and gluings along so-called $\mathcal{R}$-components, which are solid tori equipped with a 'simple' total foliation.

Section 2 is devoted to the study of the effect of a surgery on the homotopy class of a total foliation. In Subsection 2.1, we review two invariants of total plane fields that determine its homotopy class completely - the spin structure and the difference of Hopf degree. In Subsection 2.2, we define $\mathcal{R}$-components of total foliations and gluing of two total foliations along the boundaries of $\mathcal{R}$-components. In Subsections 2.3 and 2.4 , we define a surgery of a total foliation along an $\mathcal{R}$-component and give a surgery formula.

Section 3 is the main part of our construction of a total foliation in any given homotopy class. It is done by a modification of Hardorp's construction in [10]. The main new feature in our construction is a control of the framing of $\mathcal{R}$ components by insertion of 'plugs' (Lemma 3.22). Insertion of plugs of another type also enables us to control the difference of Hopf degree (Lemma 3.24). In order to obtain such plugs, we need to construct total foliations on the three-dimensional sphere $S^{3}$ such that the cores of $\mathcal{R}$-components form special framed links. Hardorp's construction is insufficient to our purpose since the framing is a very large positive number and it is difficult to control. In the first step of our construction, there are two differences from his construction :

(1) our construction is performed on a non-trivial $\mathbb{T}^{2}$-bundle over the circle while Hardorp's was on $\mathbb{T}^{3}$;

(2) foliations in our $\mathcal{R}$-components may rotate several times in some sense while they did not in Hardorp's.

These differences leads to a simpler construction in the succeeding steps: we can avoid dealing with a finite covering of a total foliation on the Poincaré sphere and with a branched double covering along the unknot. As a consequence, we can obtain an explicit description of the framings of $\mathcal{R}$-components in terms of diagrams of braids, see Proposition 3.21.

In Subsection 3.2, we give a construction of total foliations on $\mathbb{T}^{2} \times[0,1]$. In Subsection 3.4, we describe the framings of $\mathcal{R}$-components of a total foliation that 
is given by gluing two boundary components of $\mathbb{T}^{2} \times[0,1]$. In Subsection 3.5, we control the framings of $\mathcal{R}$-components and show a generalized version of Hardorp's theorem, i.e., the existence of a total foliation with any given spin structure. The control is done by successive replacements of an $\mathcal{R}$-component with a totally foliated solid torus which contains a twisted $\mathcal{R}$-component ('insertion of plugs'). In Subsection 3.6, we give a control of the Hopf degree. In fact, we construct a total foliation on $S^{3}$ that admits unknotted $\mathcal{R}$-components with $(+1)$ - and $(-1)$-framings and that has the required difference of Hopf degree with the positive total Reeb foliation. By gluing it with a total foliation that has the required spin structure, we obtain a total foliation in any given homotopy class of total plane fields.

Section 4 is devoted to the proof of Corollary 1.3. We show that if a total foliation admits an unknotted $\mathcal{R}$-component with $(+1)$-framing then any positive contact structure that is sufficiently close to one of the foliations violates the Thurston-Bennequin inequality and therefore is overtwisted. Once it is shown, the corollary is an easy consequence of Eliashberg's classification of overtwisted contact structures in [6].

1.3. Acknowledgements. This paper was prepared while the first and third authors stayed at Unité de Mathématiques Pures et Appliquées, École Normale Supérieure de Lyon and it started when the second author was at Institut Fourier, Grenoble. They thank the members of those institutions, especially Professor Étienne Ghys for his warm hospitality. The authors are also grateful to an anonymous referee for many suggestions to improve the readability of the paper.

\section{Gluing and surgery of total foliations}

2.1. Homotopy classes of plane fields. In the rest of the paper, all manifolds and foliations are of class $C^{\infty}$ and all plane fields and foliations are transversely oriented.

Fix an $n$-dimensional manifold $X$ equipped with a Riemannian metric. $\operatorname{Let} \operatorname{Fr}(X)$ be the set of orthonormal frames of $T X$. It admits a natural topology as a subset of the set of $n$-tuples of vector fields on $X$.

When $M$ is a three-dimensional manifold, by taking the unit normal vectors of a total plane field, and by applying the Gram-Schmidt orthogonalization to it, we can define a continuous map from the set of total plane fields to $\operatorname{Fr}(M)$. It is easy to see that it induces a bijection between homotopy classes. So, we consider $\operatorname{Fr}(M)$ instead of the set of total plane fields in this subsection.

First, we review some basic facts on spin structures. We denote by $\operatorname{SO}(n)$ the group of special orthogonal matrices of size $n$. Let $X$ be an $n$-dimensional manifold with $n \geq 3$. We fix a triangulation of $X$ and let $X_{i}$ be the $i$-skeleton of $X$ for $0 \leq i \leq n$. By $\operatorname{Fr}\left(X_{i}\right)$, we denote the set of orthonormal frames of $\left.T X\right|_{X_{i}}$. A spin structure is a homotopy class of $\operatorname{Fr}\left(X_{1}\right)$ of which each representative can be extended to an element of $\operatorname{Fr}\left(X_{2}\right)$. In particular, a frame $\check{e}$ in $\operatorname{Fr}(X)$ induces a spin structure on 
$X$ in a natural way. We call it the spin structure given by the frame $\check{e}$. Our definition is different from the standard one that is given by a double covering of a natural principal $\mathrm{SO}(n)$-bundle, but it is known they are equivalent if $n \geq 3$, see [11].

A manifold $X$ equipped with spin structure $s$ is called a spin manifold. If $X$ has a boundary $\partial X$, then $s$ induces a spin structure $s^{\prime}$ on $\partial X$. We call the spin manifold $\left(\partial X, s^{\prime}\right)$ the spin boundary of $(X, s)$.

Now, we focus our attention on spin structures on three or four-dimensional manifolds. We call a four-dimensional manifold $X$ a 2-handlebody if it is obtained by attaching four-dimensional 2-handles to the 4-ball $B^{4}$ along a framed link $L$ in $S^{3}=\partial B^{4}$. We say a 2-handlebody $X$ is even if the framing of each component of $L$ is even. See the first paragraph of Subsection 2.3 for the definition of framing of knots.

Proposition 2.1. Any even 2-handlebody admits a unique spin structure. Any closed spin three-dimensional manifold is a spin boundary of a spin 2-handlebody.

Proof. See Section 5.6 and 5.7 of [8].

Let $M$ be a three-dimensional closed manifold. We denote by $C(M, \mathrm{SO}(3))$ the set of continuous maps from $M$ to $\mathrm{SO}(3)$. The space $\operatorname{Fr}(M)$ of frames admits a natural action of $C(M, \mathrm{SO}(3))$ given by $(\check{e} \cdot F)(p)=\left(e^{i}(p) \cdot F(p)\right)_{i=1}^{3}$ for $\check{e}=\left(e^{i}\right)_{i=1}^{3}$ and $F \in C(M, \mathrm{SO}(3))$. We define a map $\Phi: \operatorname{Fr}(M) \times \operatorname{Fr}(M) \rightarrow C(M, \mathrm{SO}(3))$ by $\check{e}=\check{e}_{0} \cdot \Phi\left(\check{e}_{e}, \check{e}_{0}\right)$ for $\left(\check{e}_{e}, \check{e}_{0}\right) \in \operatorname{Fr}(M)^{2}$. It is easy to check that $\Phi\left(\cdot, \check{e}_{0}\right)$ is a bijective map between $\operatorname{Fr}(M)$ and $C(M, \mathrm{SO}(3))$.

We denote the field $\mathbb{Z} / 2 \mathbb{Z}$ by $\mathbb{Z}_{2}$. Recall the fundamental group $\pi_{1}(\mathrm{SO}(n))$ of $\mathrm{SO}(n)$ is isomorphic to $\mathbb{Z}_{2}$ if $n \geq 3$. Let $\operatorname{Spin}(n)$ be the universal covering group of $\mathrm{SO}(n)$.

Definition 2.2. For $\check{e}, \check{e_{0}} \in \operatorname{Fr}(M)$, we define $s\left(\check{e}, \check{e_{0}}\right) \in H^{1}\left(M, \mathbb{Z}_{2}\right)$ by

$$
s\left(\check{e}, \check{e_{0}}\right)\left([\gamma]_{H_{1}}\right)=\left[\Phi\left(\check{e}, \check{e_{0}}\right) \circ \gamma\right]_{\pi_{1}} \in \pi_{1}(\mathrm{SO}(3)) \simeq \mathbb{Z}_{2}
$$

for any continuous loop $\gamma$ in $M$. We call the above cohomology class the difference of spin structures of $\check{e}$ and $\check{e}_{0}$.

It is easy to see that $s\left(\check{e}, \check{e_{0}}\right)$ is well-defined and is determined by the homotopy classes of $\check{e}$ and $\check{e}_{0}$. We can see that $s\left(\check{e}, \check{e}_{0}\right)=0$ if and only if the restrictions of $\check{e}$ and $\check{e}_{0}$ to a fixed 1-skeleton are homotopic. In particular, $s\left(\check{e}_{,} \check{e}_{0}\right)=0$ if and only if two frames $\check{e}$ and $\check{e}_{0}$ give the same spin structure.

Lemma 2.3. If two given frames $\check{e}, \check{e}_{0} \in \operatorname{Fr}(M)$ satisfy $s\left(\check{e}_{e}, \check{e}_{0}\right)=0$, then the map $\Phi\left(\check{e}_{e}, \check{e}_{0}\right)$ admits a lift $\widetilde{\Phi}\left(\check{e}_{e}, \check{e}_{0}\right): M \rightarrow \operatorname{Spin}(3)$. 
Proof. The map $\Phi\left(\check{e}, \check{e}_{0}\right)$ induces a trivial map between the fundamental groups. Hence, it admits a lift to $\operatorname{Spin}(3)$.

Definition 2.4. When two frames $\check{e}$ and $\check{e}_{0}$ of $M$ give the same spin structure, we define the difference of Hopf degree $H\left(\check{e}, \check{e}_{0}\right)$ by the mapping degree of $\widetilde{\Phi}\left(\check{e}_{e}, \check{e}_{0}\right)$.

Remark that $H\left(\left(e^{i}\right)_{i=1}^{3},\left(e_{0}^{i}\right)_{i=1}^{3}\right)$ coincides with the difference of Hopf degree of non-singular vector fields $e^{i}$ and $e_{0}^{i}$ for any $i=1,2,3$, which is defined in [5]. It is easy to see that the formulae

$$
\begin{aligned}
& H\left(\check{e}_{2}, \check{e}_{1}\right)=H\left(-\check{e}_{1},-\check{e}_{2}\right)=-H\left(\check{e}_{1}, \check{e}_{2}\right), \\
& H\left(\check{e}_{1}, \check{e}_{3}\right)=H\left(\check{e}_{1}, \check{e}_{2}\right)+H\left(\check{e}_{2}, \check{e}_{3}\right)
\end{aligned}
$$

hold if $\check{e}_{1}, \check{e}_{2}, \check{e}_{3} \in \operatorname{Fr}(M)$ give the same spin structure, where $-\check{e}=\left(-e^{i}\right)_{i=1}^{3}$ for $\check{e}=\left(e^{i}\right)_{i=1}^{3}$.

Proposition 2.5. Two frames $\check{e}, \check{e}_{0} \in \operatorname{Fr}(M)$ are homotopic to each other if and only if they give the same spin structure and satisfy $H\left(\check{e}_{e}, \check{e}_{0}\right)=0$.

Proof. It is trivial that the former implies the latter.

Suppose the latter holds for $\check{e}_{,} \check{e}_{0} \in \operatorname{Fr}(M)$. Then, we have $s\left(\check{e}_{,}, \check{e}_{0}\right)=0$ and $H\left(\check{e}, \check{e}_{0}\right)=0$. Fix a structure of a CW complex on $M$ with a unique 3-cell. Let $M_{2}$ be the 2-skeleton of $M$. Since $\operatorname{Spin}(3)$ is homeomorphic to $S^{3}$, the lift $\widetilde{\Phi}\left(\check{e}, \check{e}_{0}\right)$ of $\Phi\left(\check{e}, \check{e}_{0}\right)$ is homotopic to a map $F$ such that $\left.F\right|_{M_{2}}$ is a constant map. Since the quotient space $M / M_{2}$ also is homeomorphic to $S^{3}$, the assumption $H\left(\check{e}, \check{e}_{0}\right)=0$ implies that $F$ is homotopic to a constant map. Therefore, $\check{e}$ is homotopic to $\check{e}_{0}$.

2.2. $\mathcal{R}$-components and gluing of total foliations. In the rest of the paper, we identify the circle $S^{1}$ with $\mathbb{R} / \mathbb{Z}$, and the two-dimensional torus $\mathbb{T}^{2}$ with $(\mathbb{R} / \mathbb{Z})^{2}$. The sum $a+b$ is well-defined for $a \in S^{1}$ and $b \in S^{1}$ or $\mathbb{R}$. For $a \in S^{1}$ and $\epsilon_{1}, \epsilon_{2} \in \mathbb{R}$, we denote the subset $\left\{a+t \in S^{1} \mid t \in\left[\epsilon_{1}, \epsilon_{2}\right]\right\}$ by $\left[a+\epsilon_{1}, a+\epsilon_{2}\right]$. We will abuse the identification of the number $t \in[0,1]$ and $t+\mathbb{Z} \in S^{1}$ when the meaning is clear.

Put $D^{2}(r)=\left\{(x, y) \in \mathbb{R}^{2} \mid x^{2}+y^{2} \leq r^{2}\right\}$ for $r \geq 0$ and $D^{2}=D^{2}(1)$. We denote $[0,1] \times \mathbb{T}^{2}$ by $W, S^{1} \times D^{2}$ by $Z$, and the origin of $\mathbb{R}^{2}$ by $O$. We also denote by $] a, b[$ the open interval $\{x \in \mathbb{R} \mid a<x<b\}$.

For a foliation $\mathscr{F}$ on a manifold $X$ and a point $p$ of $X$, let $\mathcal{F}(p)$ denote the leaf containing $p$. For a diffeomorphism $F$ from $X$ to another manifold $X^{\prime}$, let $F(\mathcal{F})$ denote a foliation on $X^{\prime}$ such that the leaf containing $F(p)$ is $F(\mathcal{F}(p))$. For a pair $\left(\mathscr{F}^{1}, \mathcal{F}^{2}\right)$ of mutually transverse codimension-one foliations on a three-dimensional manifold $M$, let $\mathscr{F}^{1} \cap \mathscr{F}^{2}$ be the one-dimensional foliation $\left\{\mathcal{F}^{1}(p) \cap \mathscr{F}^{2}(p)\right\}_{p \in M}$. 
Definition 2.6. Let $M$ be a three-dimensional manifold. We say a subset $R$ of $M$ is a thick Reeb component of a foliation $\mathscr{F}$ if $R$ contains a Reeb component $R^{\prime}$ and $\mathcal{F} \mid \overline{R \backslash R^{\prime}}$ is diffeomorphic to a product foliation $\left\{t \times \mathbb{T}^{2}\right\}_{t \in[0,1]}$ on $W$.

Let $(t, x, y)$ be the standard coordinate system of $S^{1} \times \mathbb{R}^{2}$. Take a smooth odd function $\chi_{R}$ on $\mathbb{R}$ so that $0<\chi_{R}(x)<1$ if $\left.x \in\right] 1 / 2,3 / 2\left[\right.$ and $\chi_{R}(x)=0$ otherwise. Let $\widehat{\mathcal{R}}^{1}$ and $\widehat{\mathcal{R}}^{2}$ be the foliations on $S^{1} \times \mathbb{R}^{2}$ that are generated by the kernel of $d y-\chi_{R}(y) d t$ and $d x-\chi_{R}(x) d t$, respectively.

We denote by $\mathcal{R}^{i}$ the restriction of $\hat{\mathcal{R}}^{i}$ on $Z$ for $i=1,2$. We can take a foliation $\mathcal{R}^{3}$ on $Z$ so that it is a thick Reeb component and $\left(\mathcal{R}^{i}\right)_{i=1}^{3}$ is a total foliation. See Figure 1 .
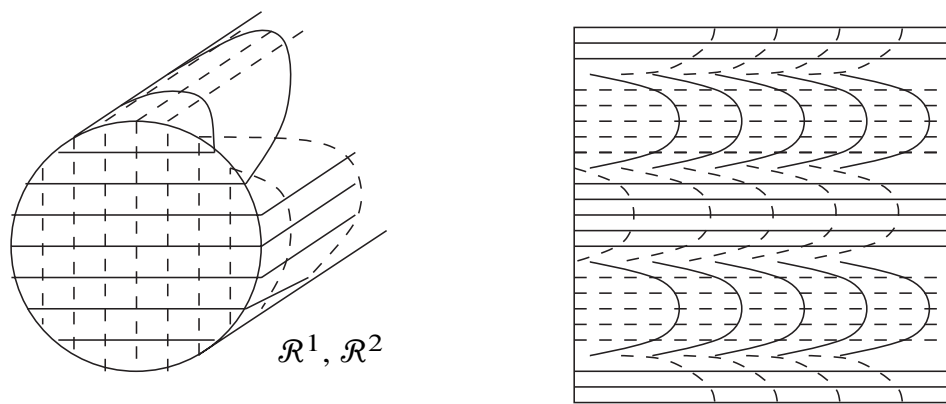

$\mathcal{R}_{T}^{1}, \mathcal{R}_{T}^{2}$

Figure 1. Foliations $\mathcal{R}^{1}, \mathcal{R}^{2}, \mathcal{R}_{T}^{1}$, and $\mathcal{R}_{T}^{2}$.

Definition 2.7. Let $\left(\mathscr{F}_{i}\right)_{i=1}^{3}$ be a total foliation on a three-dimensional manifold $M$. We call a subset $R$ of $M$ an $\mathcal{R}$-component of $\left(\mathcal{F}^{i}\right)_{i=1}^{3}$ if there exists a diffeomorphism $\psi: Z \rightarrow R$ such that $\psi\left(\mathcal{R}^{i}\right)=\left.\mathscr{F}^{i}\right|_{R}$ for $i=1,2,3$ and the restriction of $\mathscr{F}^{3}$ on a neighborhood of $\partial R$ is diffeomorphic to $\left\{t \times \mathbb{T}^{2}\right\}_{t \in[0,1]}$ on $W$. The diffeomorphism $\psi$ is called a canonical coordinate of $R$. The curve $C(R)=\psi\left(S^{1} \times 0\right)$ admits a natural orientation induced from $\psi$ and we call it the core of $R$.

Remark that the isotopy class of $C(R)$ is uniquely determined as an oriented knot in $M$.

Let $\varphi_{\mathcal{R}}: \mathbb{T}^{2} \rightarrow S^{1} \times \partial D^{2}$ be the map given by

$$
\varphi_{\mathcal{R}}(x, y)=(x, \cos (2 \pi y), \sin (2 \pi y)) .
$$

We define foliations $\mathcal{R}_{T}^{1}$ and $\mathcal{R}_{T}^{2}$ on $\mathbb{T}^{2}$ so that $\varphi_{\mathcal{R}}\left(\mathcal{R}_{T}^{i}\right)$ is the restriction of $\mathcal{R}^{i}$ on $\partial Z$ for each $i=1,2$. We use the following lemma in Section 4. 
Lemma 2.8. If a smooth line field $\xi$ on $\mathbb{T}^{2}$ is sufficiently $C^{0}$-close to $T \mathcal{R}_{T}^{1}$, then there exists a closed curve which is tangent to $\xi$ and homotopic to the curve $S^{1} \times y_{0}$, where $y_{0}$ be the point of $S^{1}$ represented by 0 .

Proof. Put $A=S^{1} \times\left[y_{0}-1 / 4, y_{0}+1 / 4\right]$. If a smooth line field $\xi$ on $\mathbb{T}^{2}$ is sufficiently $C^{0}$-close to $T \mathcal{R}_{T}^{1}$, then it is isotopic to $\partial A$ and admits an orientation which directs inward at $\partial A$. By the Poincaré-Bendixon theorem, there exists a closed curve in $A$ which is tangent to $\xi$ and isotopic to $S^{1} \times y_{0}$.

Let $a_{\mathcal{R}}$ be the integral homology class in $H_{1}\left(\mathbb{T}^{2}, \mathbb{Z}\right)$ represented by a map $x \mapsto(x, 0)$. Remark that each closed leaf of $\mathcal{R}_{T}^{1}$ is the image of a curve which represents $a_{\mathcal{R}}$.

Definition 2.9. Let $\left(\mathcal{F}^{i}\right)_{i=1}^{3}$ be a total foliation on a manifold $M$. We call a boundary component $T$ of $M$ an $\mathcal{R}$-boundary if there exists a diffeomorphism $\psi_{T}: \mathbb{T}^{2} \rightarrow T$ such that $\psi_{T}\left(\mathcal{R}_{T}^{i}\right)$ is the restriction of $\mathscr{F}^{i}$ to $T$ for $i=1,2$, and $\mathscr{F}^{3}$ is diffeomorphic to the product foliation $\left\{t \times \mathbb{T}^{2}\right\}_{t \in[0,1]}$ on a neighborhood of $T$. For an $\mathcal{R}$-boundary component $T$, we define $a_{\mathcal{R}}(T) \in H_{1}(T, \mathbb{Z})$ by $a_{\mathcal{R}}(T)=\left(\psi_{T}\right)_{*}\left(a_{\mathcal{R}}\right)$.

Remark that if $R$ is an $\mathcal{R}$-component of a total foliation on a manifold $M$, then $\partial R$ is an $\mathcal{R}$-boundary of both $R$ and $\overline{M \backslash R}$.

We define cut and paste operations of total foliations with $\mathcal{R}$-boundary by following the idea described in [10]. First, we show that the pair $\left(\left.\mathcal{F}^{1}\right|_{T},\left.\widetilde{F}^{2}\right|_{T}\right)$ of foliations of an $\mathcal{R}$-boundary of a total foliation $\left(\mathscr{F}^{i}\right)_{i=1}^{3}$ is determined by $a_{\mathcal{R}}(T)$ up to isotopy.

Lemma 2.10. Let $F$ be a diffeomorphism of $\mathbb{T}^{2}$ such that $F_{*}\left(a_{\mathcal{R}}\right)=a_{\mathcal{R}}$. Then, there exists a diffeomorphism $G$ which is isotopic to the identity and satisfies $G\left(\mathcal{R}_{T}^{i}\right)=$ $F\left(\mathcal{R}_{T}^{i}\right)$ for $i=1,2$.

Proof. Let $\tau_{y}$ be the diffeomorphism of $\mathbb{T}^{2}$ such that $\tau_{y}(x, y)=(x,-y)$. Then, $\tau_{y}\left(\mathcal{R}_{T}^{i}\right)=\mathcal{R}_{T}^{i}$ for $i=1,2$ and $\left(\tau_{y}\right)_{*}\left(a_{\mathcal{R}}\right)=a_{\mathcal{R}}$. Hence, we may assume that $F$ is orientation-preserving by replacing $F$ with $F \circ \tau_{y}$ if it is necessary.

Fix an integer $k$. Let $\tilde{h}$ be a smooth function on $\mathbb{R} \times[0,1]$ such that $\tilde{h}(y+n, t)=$ $\tilde{h}(y, t)+k n$ for any $(y, t) \in \mathbb{R} \times[0,1]$ and $n \in \mathbb{Z}$, and

$$
\tilde{h}(y, t)= \begin{cases}k n & \text { if } y \in[n, n+(1 / 32)], \\ k(n+t) & \text { if } y \in[n+(1 / 16), n+(1 / 4)], \\ k(n+1) & \text { if } y \in[n+(9 / 32), n+1]\end{cases}
$$

for any $n \in \mathbb{Z}$ and $t \in[0,1]$. See Figure 2. The function $\tilde{h}$ induces a map $h: S^{1} \times$ $[0,1] \rightarrow S^{1}$. Remark that $h(\cdot, t): S^{1} \rightarrow S^{1}$ is a map of degree $k$.

For $t \in[0,1]$, we define a diffeomorphism $F_{k, t}$ of $\mathbb{T}^{2}$ by

$$
F_{k, t}(x, y)=(x+h(y, t), y) .
$$


Since $F$ is orientation-preserving and $F_{*}\left(a_{\mathcal{R}}\right)=a_{\mathcal{R}}, F$ is isotopic to $F_{k, 1}$ for some $k \in \mathbb{Z}$. Hence, it is sufficient to show that there exists a diffeomorphism $G$ of $\mathbb{T}^{2}$ which is isotopic to the identity and satisfies $G\left(\mathcal{R}_{T}^{i}\right)=F_{k, 1}\left(\mathcal{R}_{T}^{i}\right)$ for $i=1,2$.
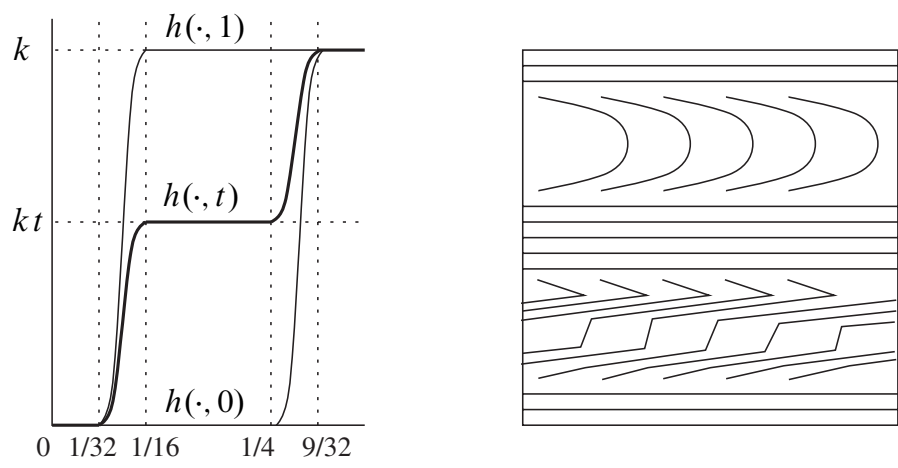

Figure 2. The map $h(\cdot, t)$ and the foliation $F_{k, 1}\left(\mathcal{R}^{1}\right)$ for $k=1$.

Since $\mathcal{R}_{T}^{1}\left(x, y_{1}\right)=S^{1} \times y_{1}$ for $y_{1} \in[0,1 / 16]$ and $\mathcal{R}_{T}^{2}\left(x, y_{2}\right)=S^{1} \times y_{2}$ for $y_{2} \in[1 / 4,5 / 16]$, we have $F_{k, 0}\left(\mathcal{R}_{T}^{2}\right)=\mathcal{R}_{T}^{2}$ and $F_{k, 1}\left(\mathcal{R}_{T}^{1}\right)=\mathcal{R}_{T}^{1}$. The foliations $\mathcal{R}_{T}^{1}$ and $\mathcal{R}_{T}^{2}$ are invariant under the translation $(x, y) \mapsto(x+t, y)$. It implies that $F_{k, t}\left(\mathcal{R}_{T}^{2}\right)$ is transverse to $\mathcal{R}_{T}^{1}$ for any $t \in[0,1]$. We define an isotopy $\left\{G_{t}\right\}_{t \in[0,1]}$ by $G_{t}(x, y) \in \mathcal{R}_{T}^{1}(x, y) \cap F_{k, t}\left(\mathcal{R}_{T}^{2}(x, y)\right)$. Then, the map $G_{0}$ is the identity, $G_{1}\left(\mathcal{R}_{T}^{1}\right)=\mathcal{R}_{T}^{1}=F_{k, 1}\left(\mathcal{R}_{T}^{1}\right)$, and $G_{1}\left(\mathcal{R}_{T}^{2}\right)=F_{k, 1}\left(\mathcal{R}_{T}^{2}\right)$.

Proposition 2.11. For $k=1,2$, let $M_{k}$ be a three-dimensional manifold with a toral boundary $T_{k}$ and $\left(\mathcal{F}_{k}^{i}\right)_{i=1}^{3}$ a total foliation of $M_{k}$ such that $T_{k}$ is an $\mathcal{R}$-boundary. Suppose that a diffeomorphism $\psi: T_{1} \rightarrow T_{2}$ satisfies $\psi_{*}\left(a_{\mathcal{R}}\left(T_{1}\right)\right)=a_{\mathcal{R}}\left(T_{2}\right)$. Then, there exists a total foliation $\left(\mathcal{F}^{i}\right)_{i=1}^{3}$ on $M_{1} \cup_{\psi} M_{2}=M_{1} \cup M_{2} /[p \sim \psi(p)]$ and diffeomorphisms $F_{1}: M_{1} \rightarrow M_{1}$ and $F_{2}: M_{2} \rightarrow M_{2}$ such that $F_{k}$ is isotopic to the identity and $F_{k}\left(\mathcal{F}_{k}^{i}\right)=\left.\mathscr{F}^{i}\right|_{M_{k}}$ for any $i=1,2,3$ and $k=1,2$.

Proof. By Lemma 2.10, we can isotope $\left(\mathcal{F}_{2}^{i}\right)_{i=1}^{3}$ so that it is compatible with $\left(\mathcal{F}_{1}^{i}\right)_{i=1}^{3}$ on a neighborhood of $T_{1}=T_{2}$ in $M_{1} \cup_{\psi} M_{2}$.

2.3. Knotted $\mathcal{R}$-components and surgery. Let $M$ be an oriented three-dimensional manifold. For a smooth link $L$ in $M$, let $\operatorname{Fr}(L ; M)$ be the set of vector fields $v: L \rightarrow T M$ on $L$ satisfying $v(p) \notin T_{p} L$ for any $p \in L$. A framing of $L$ is a connected component of $\operatorname{Fr}(L ; M)$. An oriented knot $K$ is null-homologous if and only if it admits a Seifert surface $S$, that is, an oriented embedded surface with $\partial S=K$. 
Definition 2.12. Suppose an oriented knot $K$ admits a Seifert surface $S$. We call an orientation preserving embedding $\psi: S^{1} \times D^{2} \rightarrow M$ an $n$-framed tubular coordinate of $K$ if the restriction of $\psi$ to $S^{1} \times\{(0,0)\}$ is an orientation preserving diffeomorphism onto $K$ and the algebraic intersection number of $S$ and $\psi\left(S^{1} \times\{(1,0)\}\right)$ is $n$. The framing represented by a vector field $v \in \operatorname{Fr}(K ; M)$ tangent to $\psi\left(S^{1} \times[-1,1] \times\{0\}\right)$ is called an $n$-framing of $K$.

It is known that the $n$-framing of $K$ does not depend on the choices of $S$ and $\psi$.

If a link $L$ is tangent to leaves of a foliation $\mathcal{F}$, then a vector field $v_{L}$ on $L$ with $v_{L}(p) \in T_{p} \mathscr{F} \backslash T_{p} L$ gives a framing of $L$. We call it the framing given by $\mathscr{F}$. We say an $\mathcal{R}$-component $R$ of a total foliation $\left(\mathscr{F}^{i}\right)_{i=1}^{3}$ on $M$ is null-homotopic if the core $C(R)$ is null-homotopic. In addition, if $\mathscr{F}^{1}$ gives the $n$-framing of $C(R)$, we say that $R$ is an $n$-framed null-homotopic $\mathcal{R}$-component. A knot is called unknotted if it bounds an embedded disk. We say an $\mathcal{R}$-component of a total foliation on $M$ is unknotted if the core is unknotted.

Suppose that a total foliation $\left(\mathcal{F}^{i}\right)_{i=1}^{3}$ on $M$ admits an $\mathcal{R}$-component $R$. Let $\mu(R) \in H_{1}(\partial R, \mathbb{Z})$ be the homology class represented by a meridian of $R$. Up to isotopy, there exists a unique diffeomorphism $F$ on $\partial R$ such that $F_{*}\left(a_{\mathcal{R}}(\partial R)\right)=a_{\mathcal{R}}(\partial R)$ and $F_{*}(\mu(R))=\mu(R)+a_{\mathcal{R}}(\partial R)$. We call $M_{R}=(\overline{M \backslash R} \cup R) / F(p) \sim p$ the manifold obtained by the standard surgery along $R$. By Proposition 2.11, total foliations $\left(\left.\mathscr{F}^{i}\right|_{\bar{M} \backslash R}\right)_{i=1}^{3}$ and $\left(\left.\mathscr{F}^{i}\right|_{R}\right)_{i=1}^{3}$ induce a total foliation $\left(\mathcal{F}_{F}^{i}\right)_{i=1}^{3}$. We call $\left(\mathcal{F}_{F}^{i}\right)_{i=1}^{3}$ the total foliation obtained by the standard surgery along $R$. In [10], p. 22-24, one can see another surgery along an $\mathcal{R}$-component, which essentially yields the same total foliation.

Lemma 2.13. If $R$ is null-homotopic and $k$-framed, then the above $M_{R}$ is a manifold obtained by a Dehn surgery along $C(R)$ with framing coefficient $k+1$.

Proof. Since $R$ is $k$-framed, $\lambda(R)=a_{\mathcal{R}}(\partial R)-k \mu(R)$ is represented by the longitude of $C(R)$ corresponding to the 0 -framing. The condition $F_{*}(\mu(R))=\lambda(R)+$ $(k+1) \mu(R)$ implies that the coefficient of the Dehn surgery is $k+1$.

Let $\left(\mathscr{F}^{i}\right)_{i=1}^{3}$ be a total foliation on $S^{3}$ and $R_{1}, \ldots, R_{k}$ be its $\mathcal{R}$-components with the $n_{1}, \ldots, n_{k}$-framings. Lemma 2.13 implies that the manifold obtained by the standard surgery along $\mathcal{R}$-components $R_{1}, \ldots, R_{k}$ is the boundary of the fourdimensional 2-handlebody $X$ whose Kirby diagram is $\bigcup_{j=1}^{k} C(R)$ with the $\left(n_{j}+1\right)$-framing on each $C\left(R_{j}\right)$.

As we saw in Subsection 2.1, each total plane field on $M$ defines a spin structure on $M$. For a total foliation $\left(\mathcal{F}^{i}\right)_{i=1}^{3}$, we say a spin structure on $M$ is given by $\left(\mathcal{F}^{i}\right)_{i=1}^{3}$ if it is given by the total plane field $\left(T \mathscr{F}^{i}\right)_{i=1}^{3}$.

Let $\left(\mathcal{F}_{0}^{i}\right)_{i=1}^{3}$ be a total foliation on $S^{3}$ with odd-framed $\mathcal{R}$-components $R_{1}, \ldots, R_{k}$. Let $M$ and $\left(\mathscr{F}^{i}\right)_{i=1}^{3}$ denote the three-dimensional manifold and the total foliation obtained by the standard surgeries on all $R_{i}$ 's, and $X$ the four-dimensional 2-handlebody 
corresponding to the surgery as above. By Proposition 2.1, $X$ admits a unique spin structure $s_{X}$.

Proposition 2.14. The restriction of $s_{X}$ to $M=\partial X$ coincides with the one given by $\left(\mathcal{F}^{i}\right)_{i=1}^{3}$.

Proof. Let $h_{j} \subset X$ be the 2-handles corresponding to $C\left(R_{j}\right)$ for $j=1, \ldots, k$. Total foliations $\left(\mathscr{F}_{0}^{i}\right)_{i=1}^{3}$ and $\left(\mathscr{F}^{i}\right)_{i=1}^{3}$ define a spin structure $s_{*}$ on a neighborhood of $S^{3} \cup M=\partial D^{4} \cup \partial X$ in $X=D^{4} \cup \bigcup_{j=1}^{k} h_{j}$, where $D^{4}$ is the four-dimensional ball. Since $H_{1}\left(S^{3}, \mathbb{Z}_{2}\right)=0$, the sphere $S^{3}$ admits a unique spin structure. It is known that it extends to $D^{4}$. The closure of a connected component of $X \backslash\left(S^{3} \cup M\right)$ is either the ball $D^{4}$ or a 2-handle $h_{j}$. Since they are homeomorphic to the four dimensional ball, the spin structure on $S^{3} \cup M$ can be extended to $X$. By the uniqueness of a spin structure on a 2-handlebody, it completes the proof.

2.4. Gluing formula of the difference of Hopf invariant. For two total foliations $\left(\mathcal{F}^{i}\right)_{i=1}^{3}$ and $\left(\mathscr{E}^{i}\right)_{i=1}^{3}$ which give the same spin structure, we denote the difference of Hopf invariant of the corresponding orthonormal frames (see Definition 2.4) by $H\left(\left(\mathcal{F}^{i}\right)_{i=1}^{3},\left(\mathscr{G}^{i}\right)_{i=1}^{3}\right)$.

Definition 2.15. The positive total Reeb foliation $\left(\mathcal{R}_{+}^{i}\right)_{i=1}^{3}$ is a total foliation on $S^{3}$ which is the union of two $(-1)$-framed unknotted $\mathcal{R}$-components.

Remark that each $\mathcal{R}_{+}^{i}$ is a thick Reeb foliation and the cores of two $\mathcal{R}$-components form a positive ${ }^{1}$ Hopf link under the transverse orientation of $\mathcal{R}_{+}^{3}$.

Let $\tau_{S^{3}}$ be an orientation reversing diffeomorphism on $S^{3}$. It is known that $H\left(\left(\mathcal{R}_{+}^{i}\right)_{i=1}^{3}, \tau_{S^{3}}\left(\mathcal{R}_{+}^{i}\right)_{i=1}^{3}\right)=1$ (see e.g. Lemma 24 in [4]). By formulae (1) and (2) on page 276 , we have

$$
H\left(\left(\tau_{S^{3}}\left(\mathcal{F}^{i}\right)\right)_{i=1}^{3},\left(\mathcal{R}_{+}^{i}\right)_{i=1}^{3}\right)=-1-H\left(\left(\mathcal{F}^{i}\right)_{i=1}^{3},\left(\mathcal{R}_{+}^{i}\right)_{i=1}^{3}\right),
$$

for any total foliation $\left(\mathscr{F}^{i}\right)_{i=1}^{3}$ on $S^{3}$.

Let $\left(\mathscr{F}^{i}\right)_{i=1}^{3}$ and $\left(\mathscr{E}^{i}\right)_{i=1}^{3}$ be total foliations on $M$ and $S^{3}$, respectively. Suppose that $\left(\mathcal{F}^{i}\right)_{i=1}^{3}$ admits a null-homotopic $\mathcal{R}$-component $R$ and $\left(\mathscr{E}^{i}\right)_{i=1}^{3}$ admits a (-1)-framed unknotted $\mathcal{R}$-component $R^{\prime}$. Since both $R$ and $\overline{S^{3} \backslash R^{\prime}}$ are diffeomorphic to $S^{1} \times D^{2}$, there exists a diffeomorphism $\psi: \overline{S^{3} \backslash R^{\prime}} \rightarrow R$ such that $\psi_{*}\left(a_{\mathcal{R}}\left(\partial R^{\prime}\right)\right)=a_{\mathcal{R}}(\partial R)$ and $\psi_{*}\left(\mu_{R^{\prime}}\right)=\mu_{R}$. Remark that the isotopy class of $\psi$ is uniquely determined. By Proposition 2.11, there exists a total foliation $\left(\mathcal{F}^{i} \cup_{R, R^{\prime}} \mathscr{G}^{i}\right)_{i=1}^{3}$ on $M$ such that it coincides with $\left(\mathscr{F}^{i}\right)_{i=1}^{3}$ on $\overline{M \backslash R}$ and with $\left(\psi\left(\mathscr{E}^{i}\right)\right)_{i=1}^{3}$ on $R$ up to isotopy.

\footnotetext{
${ }^{1}$ Such a Reeb foliation is called a positive Reeb foliation. The orientations given as the core of the $\mathcal{R}$ component and given by the transverse orientation of $\mathcal{R}_{0}^{3}$ are opposite on one of the cores.
} 
Proposition 2.16. In the above situation, we have

$$
H\left(\left(\mathcal{F}^{i} \cup_{R, R^{\prime}} \mathscr{E}^{i}\right)_{i=1}^{3},\left(\mathcal{F}^{i}\right)_{i=1}^{3}\right)=H\left(\left(\mathscr{E}^{i}\right)_{i=1}^{3},\left(\mathcal{R}_{+}^{i}\right)_{i=1}^{3}\right)
$$

Proof. First, we notice that if two frames $\check{e}$ and $\check{e}$ on a three-dimensional manifold $M^{\prime}$ gives the same spin structure, then $H\left(\check{e}, \check{e}^{\prime}\right)$ is equal to the algebraic intersection number of the submanifolds $\left\{\check{e}(p) \mid p \in M^{\prime}\right\}$ and $\left\{-\check{e}^{\prime}(p) \mid p \in M^{\prime}\right\}$ of the orthonormal frame bundle of $M^{\prime}$.

For convenience, fix Riemannian metrics on $M$ and $S^{3}$ so that $\psi$ is an isometry between $\overline{S^{3} \backslash R^{\prime}}$ and $R$. Let $\check{e}_{\mathcal{F}}, \check{e}_{\mathscr{E}}, \check{e}_{\mathcal{R}}, \check{e}_{*}$ be the orthonormal frames induced from $\left(\mathcal{F}^{i}\right)_{i=1}^{3},\left(\mathscr{G}^{i}\right)_{i=1}^{3},\left(\mathcal{R}_{+}^{i}\right)_{i=1}^{3}$, and $\left(\mathscr{F}^{i} \cup_{R, R^{\prime}} \mathscr{G}^{i}\right)_{i=1}^{3}$, respectively. By modifying $\left(\mathcal{R}_{+}^{i}\right)_{i=1}^{3}$ in its isotopy class, we may assume that $R^{\prime}$ is a $(-1)$-framed $\mathcal{R}$-component of $\left(\mathcal{R}_{+}^{i}\right)_{i=1}^{3}$ and $\psi\left(\left.\check{e}_{\mathcal{R}}\right|_{S^{3} \backslash R^{\prime}}\right)=\left.\check{e}_{\mathcal{F}}\right|_{R}$. Take submanifolds $\Lambda=\left\{\check{e}_{\mathscr{E}}(p) \mid p \in S^{3}\right\}$ and $\Lambda^{\prime}=\left\{-\check{e}_{\mathcal{R}}(p) \mid p \in S^{3}\right\}$ of the orthonormal frame bundle of $S^{3}$. Let Fr $\psi$ be the map between the frame bundles on $\overline{S^{3} \backslash R^{\prime}}$ and $R$ induced by $\psi$. Then, we have $\operatorname{Fr} \psi(\Lambda)=\left\{\check{e}_{*}(p) \mid p \in R\right\}$ and $\operatorname{Fr} \psi\left(\Lambda^{\prime}\right)=\left\{-\check{e}_{\mathscr{F}}(p) \mid p \in R\right\}$. Since $\left.\left(\mathcal{F}^{i} \cup_{R, R^{\prime}} \mathcal{E}^{i}\right)\right|_{\overline{M \backslash R}}=\left.\mathcal{F}^{i}\right|_{\overline{M \backslash R}}$, we also have

$$
\operatorname{Fr} \psi\left(\Lambda \cap \Lambda^{\prime}\right)=\left\{\check{e}_{*}(p) \mid p \in M\right\} \cap\left\{-\check{e}_{\mathcal{F}}(p) \mid p \in M\right\} .
$$

This implies formula (4).

\section{Construction of total foliations}

3.1. Braids in $\boldsymbol{W}$. Let $\operatorname{SL}(2, \mathbb{Z})$ denote the group of $2 \times 2$-integer matrices with determinant one, and $I$ denote the identity matrix in $\operatorname{SL}(2, \mathbb{Z})$. Each element $A$ of $\operatorname{SL}(2, \mathbb{Z})$ acts on $\mathbb{T}^{2}$ as a diffeomorphism.

Fix $n \geq 1$ and define the points $Q_{j}=(j / n, j / n)+\mathbb{Z}^{2} \in \mathbb{T}^{2}$ for $j=0, \ldots, n-1$.

Definition 3.1. For $A \in \operatorname{SL}(2, \mathbb{Z})$ and $n \geq 1$, we say $\Gamma \subset[0,1] \times \mathbb{T}^{2}$ is a smooth $n$-braid twisted by $A$ if there exists a map $\gamma:\{0, \ldots, n-1\} \times[0,1] \rightarrow \mathbb{T}^{2}$ and a permutation $\sigma$ on $\{0, \ldots, n-1\}$ such that

- $\Gamma=\{(t, \gamma(j, t)) \mid(j, t) \in\{0, \ldots, n-1\} \times[0,1]\}$.

- $\gamma(j, t) \neq \gamma\left(j^{\prime}, t\right)$ for any $t \in[0,1]$ if $j \neq j^{\prime}$, and

- $\gamma(j, \varepsilon)=Q_{j}$ and $\gamma(j, 1-\varepsilon)=A \cdot Q_{\sigma(j)}$ for any $j=0, \ldots, n-1$ and any sufficiently small $\varepsilon \geq 0$.

We call a subset $\Gamma^{j}=\{(t, \gamma(j, t)) \mid t \in[0,1]\}$ the $j$-th string of $\Gamma$.

Let $B_{n}(A)$ be the set of all smooth $n$-braids twisted by $A$. 
We can identify $B_{n}(A)$ with a set of smooth maps from $\{0, \ldots, n-1\} \times[0,1]$ to $\mathbb{T}^{2}$. This identification induces a topology on $B_{n}(A)$. Let $\pi_{0}\left(B_{n}(A)\right)$ be the set of connected components of $B_{n}(A)$.

For $A \in \mathrm{SL}(2, \mathbb{Z})$, let $F_{A}$ be the diffeomorphism on $W$ given by $F_{A}(t, w)=$ $(t, A \cdot w)$. We define $\tau_{1}(t, w)=(1-t, w), \tau_{-}(t, w)=(t / 2, w)$, and $\tau_{+}(t, w)=$ $((1+t) / 2, w)$ for $(t, w) \in W=[0,1] \times \mathbb{T}^{2}$.

Definition 3.2. Let $\Gamma$ be a braid in $B_{n}(A)$.

- The inverse $\Gamma^{-1} \in B_{n}\left(A^{-1}\right)$ is defined by $\Gamma^{-1}=F_{A^{-1}} \circ \tau_{1}(\Gamma)$.

- The composition $\Gamma * \Gamma^{\prime} \in B_{n}\left(A^{\prime} \cdot A\right)$ for $\Gamma \in B_{n}(A)$ and $\Gamma^{\prime} \in B_{n}\left(A^{\prime}\right)$ is defined by $\Gamma * \Gamma^{\prime}=\tau_{-}(\Gamma) \cup\left(F_{A} \circ \tau_{+}\right)\left(\Gamma^{\prime}\right)$.

They induce corresponding operations on $\pi_{0}\left(B_{n}(A)\right)$. We can see that they define a group structure on $\pi_{0}\left(B_{n}(I)\right)$, which is isomorphic to the braid group of $n$-strings on $\mathbb{T}^{2}$. The composition also defines a free and transitive action of $\pi_{0}\left(B_{n}(I)\right)$ on $\pi_{0}\left(B_{n}(A)\right)$. In particular, each element of $\pi_{0}\left(B_{n}(A)\right)$ gives a bijective map between $\pi_{0}\left(B_{n}(I)\right)$ and $\pi_{0}\left(B_{n}(A)\right)$.

3.2. Total foliations with braided leaves. In this subsection, we fix an integer $n \geq 1$ and a real number $\eta>0$ which is sufficiently smaller than $1 / n$, for example, $\eta=(100 n)^{-1}$. Put $q_{j}=(j / n)+\mathbb{Z} \in S^{1}$ for $j=0, \ldots, n-1$. Recall that $Q_{j}=\left(q_{j}, q_{j}\right) \in \mathbb{T}^{2}$.

First, we define the standard total foliation $\left(\mathcal{F}_{\text {std }}^{i}\right)_{i=1}^{3}$ on $W=[0,1] \times \mathbb{T}^{2}$. Let $(t, x, y)$ be the standard coordinate system of $W=[0,1] \times \mathbb{T}^{2}$. Fix a smooth function $\bar{\chi}_{1}$ on $\mathbb{R}$ such that $0<\bar{\chi}_{1}(x)<\eta$ for $\left.x \in\right] 1 / 16 n, 1 / 8 n\left[\right.$ and $\bar{\chi}_{1}(x)=0$ otherwise. Let $\chi_{1}$ be the function on $S^{1}$ given by $\chi_{1}\left(q_{j}+x\right)=\bar{\chi}_{1}((1 / 2 n)+x)-\bar{\chi}_{1}((1 / 2 n)-x)$ for any $j=0, \ldots, n-1$ and $x \in[0,1 / n]$. See Figure 3. We define 1 -forms $\omega_{\text {std }}^{1}$,

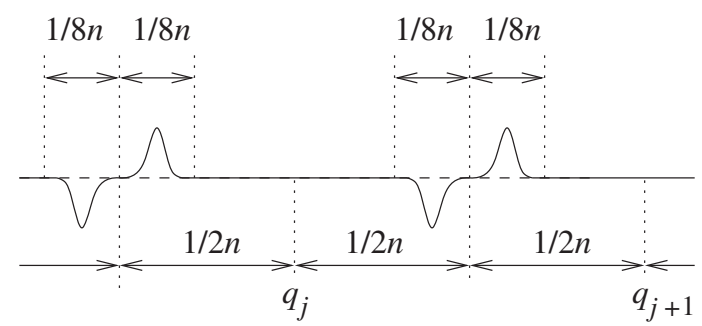

Figure 3. Function $\chi_{1}$ 
$\omega_{\text {std }}^{2}$, and $\omega_{\text {std }}^{3}$ on $W$ by

$$
\begin{aligned}
& \omega_{\text {std }}^{1}(t, x, y)=d y-\chi_{1}(y) d x, \\
& \omega_{\text {std }}^{2}(t, x, y)=d x-\chi_{1}(x) d y, \\
& \omega_{\text {std }}^{3}(t, x, y)=d t-\left(\bar{\chi}_{1}(t-3 / 8)+\bar{\chi}_{1}(t-5 / 8)\right) d y .
\end{aligned}
$$

Let $\mathcal{F}_{\text {std }}^{i}$ be the foliation generated by the kernel of $\omega_{\text {std }}^{i}$ for $i=1,2,3$. See Figure 4. It is easy to check that the triple $\left(\mathcal{F}_{\text {std }}^{i}\right)_{i=1}^{3}$ is a total foliation.
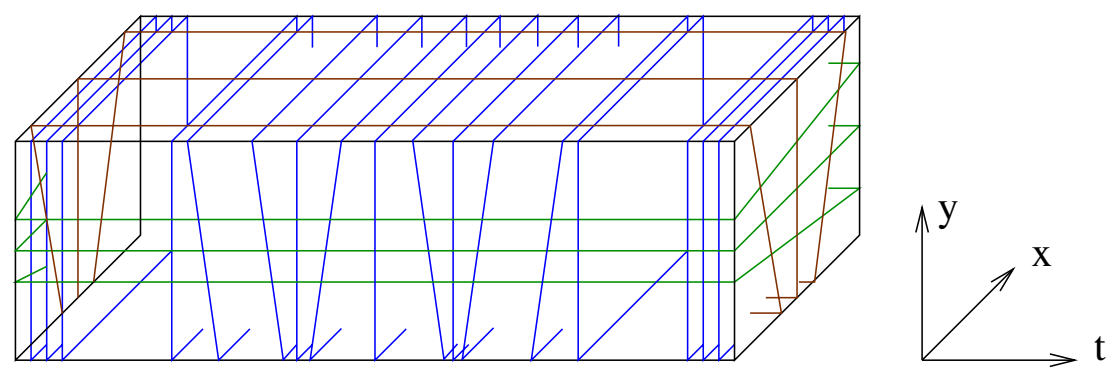

Figure 4. Total foliation $\left(\mathcal{F}_{\text {std }}^{i}\right)_{i=1}^{3}$ on $[0,1] \times[j / n,(j+1) / n]^{2}$.

Definition 3.3. Let $R$ be an embedded solid torus in $M$ and $\mathcal{F}$ a foliation on $\overline{M \backslash R}$. We say a foliation $\mathcal{F}_{*}$ is obtained by a turbularization of $\mathcal{F}_{\text {along }} R$ if $R$ is a thick Reeb component of $\mathscr{F}_{*}$ and there exists a diffeomorphism $\psi$ of the open manifold $M \backslash R$ which is isotopic to the identity and satisfies $\left.\mathcal{F}_{*}\right|_{M \backslash R}=\psi(\mathcal{F})$.

Observe that if the restriction of $\mathcal{F}$ to $R$ is isotopic to the product foliation $\left\{\{\mathrm{pt}\} \times D^{2}\right\}$, then we can turbularize $\mathcal{F}$ along $R$.

Let $U_{j}$ be the interior of $[1 / 4,1 / 3] \times\left[q_{j}+(1 / 4 n), q_{j}+(3 / 4 n)\right] \times S^{1}$ for $j=0, \ldots, n-1$ and

$$
W_{0}=W \backslash \bigcup_{j=0}^{n-1} U_{j}
$$

Definition 3.4. We say a foliation $\widetilde{F}_{0}$ on a subset $W^{\prime}$ of $W$ is almost horizontal if

$$
T \mathcal{F}_{0}(p) \subset\left\{v \in T_{p} W^{\prime} \mid d y(v)^{2} \leq \eta^{-2}\left(d t(v)^{2}+d x(v)^{2}\right)\right\}
$$

for any $p \in W^{\prime}$.

The next proposition shows how to make almost horizontal foliations part of a total foliation. 
Proposition 3.5. For any given almost horizontal foliation $\mathcal{F}$ on $W_{0}$, there exists an extension $\mathscr{F}^{1}$ of $\mathcal{F}$ to $W$ such that $\left(\mathcal{F}^{1}, \widetilde{F}_{\text {std }}^{2}, \widetilde{F}_{\text {std }}^{3}\right)$ is a total foliation.

Proof. Put

$$
\begin{aligned}
& R_{j}^{+}=\left\{\left((3 / 8)+t, q_{j}+(1 / 2 n)+x\right) \mid(t, x) \in D^{2}(1 / 8 n)\right\} \times S^{1}, \\
& R_{j}^{-}=\left\{\left((5 / 8)+t, q_{j}+(1 / 2 n)+x\right) \mid(t, x) \in D^{2}(1 / 8 n)\right\} \times S^{1}
\end{aligned}
$$

for $j=0, \ldots, n-1$. Let $f_{j}$ be a diffeomorphism of $S^{1}$ which is conjugate to the holonomy map of $F^{F}$ along the torus $\partial U_{j}$. By $r_{\alpha}$, we denote the rigid rotation of

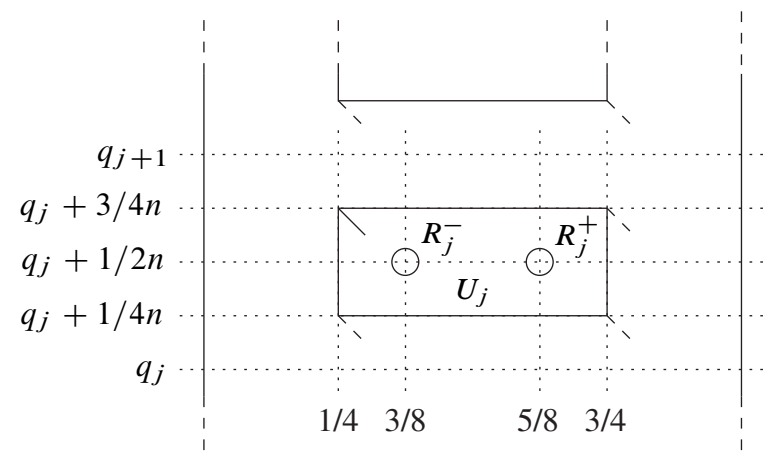

Figure 5. The sets $W_{0}$ and $R_{j}^{ \pm}$.

angle $\alpha \in \mathbb{R}$, i.e., $r_{\alpha}(y)=y+\alpha$. By a consequence of the Fundamental Theorem of Herman (see e.g. [3], Corollary 8.5.3), there exist $\alpha_{j}^{-}, \alpha_{j}^{+} \in \mathbb{R}$ and a diffeomorphism $g_{j}$ on $S^{1}$ such that $f_{j}=\left(g_{j} \circ r_{\alpha_{j}^{-}} \circ g_{j}^{-1}\right) \circ r_{\alpha_{j}^{+}}$for any $j=0, \ldots, n-1$. It implies that we can extend $\mathcal{F}$ to an almost horizontal foliation $\mathscr{E}$ on $\overline{W \backslash \bigcup_{j=0}^{n-1}\left(R_{j}^{-} \cup R_{j}^{+}\right)}$such that the holonomy map of $\mathscr{E}$ along the torus $\partial R_{j}^{\sigma}$ is conjugate to the rigid rotation $r_{\alpha_{j}^{\sigma}}$ for any $j=0, \ldots, n-1$ and $\sigma= \pm$. Since $\mathscr{E}$ is almost horizontal, it is transverse to $\mathcal{F}_{\text {std }}^{2}$ and $\mathcal{F}_{\text {std }}^{3}$. A turbularization of $\mathscr{E}$ along all $R_{j}^{ \pm}$gives a foliation $\mathcal{F}^{1}$ on $W$ which is transverse to both $\mathcal{F}_{\text {std }}^{2}$ and $\mathcal{F}_{\text {std. }}^{3}$. See Figure 6 .

Recall that $F_{A}(t, w)=(t, A w), \tau_{1}(t, w)=(1-t, w), \tau_{-}(t, w)=(t / 2, w)$, and $\tau_{+}(t, w)=((t+1) / 2, w)$ for $A \in \operatorname{SL}(2, \mathbb{Z})$ and $(t, w) \in W$. Let $\left(e_{t}, e_{x}, e_{s}\right)$ be the orthonormal frame on $W$ which corresponds to the standard coordinates $(t, x, y)$.

Definition 3.6. For $A \in \operatorname{SL}(2, \mathbb{Z})$, let $\operatorname{tFol}(A)$ be the set of total foliations $\left(\mathcal{F}^{i}\right)_{i=1}^{3}$ on $W$ such that

- $\mathcal{F}^{3}$ is transverse to $e_{t}$.

- $\mathscr{F}^{i}=\mathscr{F}_{\text {std }}^{i}$ on a neighborhood of $\{0\} \times \mathbb{T}^{2}$ for $i=1,2,3$,

- $\mathscr{F}^{i}=F_{A}\left(\mathscr{F}_{\text {std }}^{i}\right)$ on a neighborhood of $\{1\} \times \mathbb{T}^{2}$ for $i=1,2,3$ 

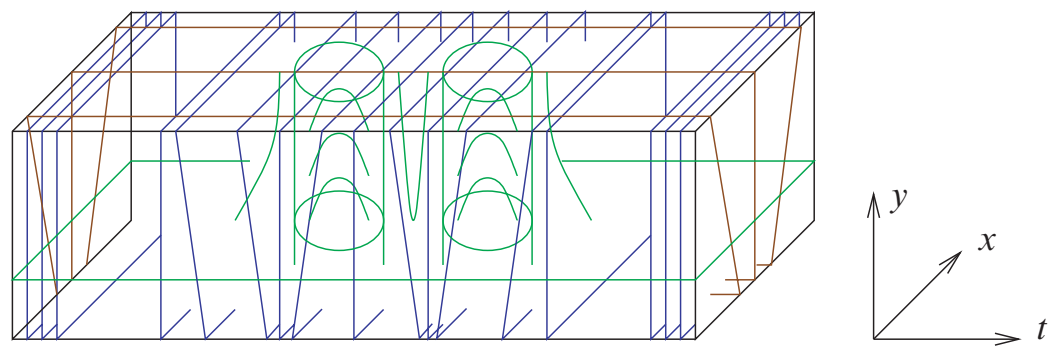

Figure 6. Total foliation associated to an extension of $\mathscr{F}_{0}$.

We introduce some operations on total foliations in $\operatorname{tFol}(A)$.

Definition 3.7. Let $\left(\mathcal{F}^{i}\right)_{i=1}^{3}$ and $\left(\mathscr{G}^{i}\right)_{i=1}^{3}$ be total foliations in $\operatorname{tFol}(A)$ and $\operatorname{tFol}\left(A^{\prime}\right)$ respectively.

- The inverse $\left(\left(\mathcal{F}^{i}\right)^{-1}\right)_{i=1}^{3} \in \operatorname{tFol}\left(A^{-1}\right)$ is defined by $\left(\mathcal{F}^{i}\right)^{-1}=F_{A^{-1}} \circ \tau_{1}\left(\mathcal{F}^{i}\right)$ for $i=1,2,3$.

- The composition $\left(\mathcal{F}^{i} * \mathscr{E}^{i}\right)_{i=1}^{3} \in \operatorname{tFol}\left(A^{\prime} A\right)$ of $\left(\mathcal{F}^{i}\right)_{i=1}^{3}$ and $\left(\mathscr{E}^{i}\right)_{i=1}^{3}$ is defined by $\left.\left(\mathcal{F}^{i} * \mathscr{E}^{i}\right)\right|_{[0,1 / 2]}=\tau_{-}\left(\mathcal{F}^{i}\right)$ and $\left.\left(\mathscr{F}^{i} * \mathscr{E}^{i}\right)\right|_{[1 / 2,1]}=\left(\tau_{+} \circ F_{A}\right)\left(\mathscr{E}^{i}\right)$.

We define an important subset of $\operatorname{tFol}(A)$ consisting of total foliations with braided leaves.

Definition 3.8. For $A \in \operatorname{SL}(2, \mathbb{Z})$, we denote by $\operatorname{tFol}(A, n)$ the subset of $\operatorname{tFol}(A)$ consisting of total foliations $\left(\mathcal{F}^{i}\right)_{i=1}^{3}$ such that $\Gamma=\bigcup_{j=0}^{n-1}\left(\mathcal{F}^{1} \cap \mathcal{F}^{2}\right)\left(0, Q_{j}\right)$ is an element of $B_{n}(A)$. For $\left(\mathcal{F}^{i}\right)_{i=1}^{3} \in \operatorname{tFol}(A, n)$, we denote the connected component of $B_{n}(A)$ containing the above $\Gamma$ by $\sigma\left(\left(\mathcal{F}^{i}\right)_{i=1}^{3}\right)$.

For any given $\left(\mathcal{F}^{i}\right)_{i=1}^{3} \in \operatorname{tFol}(A, n)$ and $\left(\mathscr{E}^{i}\right)_{i=1}^{3} \in \operatorname{tFol}\left(A^{\prime}, n\right)$, it is easy to verify that $\left(\left(\mathcal{F}^{i}\right)^{-1}\right)_{i=1}^{3}$ is an element of $\operatorname{tFol}\left(A^{-1}, n\right)$ with $\sigma\left(\left(\left(\mathcal{F}^{i}\right)^{-1}\right)_{i=1}^{3}\right)=$ $\sigma\left(\left(\widetilde{F}^{i}\right)_{i=1}^{3}\right)^{-1}$ and $\left(\tilde{F}^{i} * \mathscr{E}^{i}\right)_{i=1}^{3}$ is an element of $\mathrm{tFol}\left(A^{\prime} A, n\right)$ with $\sigma\left(\left(\mathcal{F}^{i} * \mathscr{G}^{i}\right)_{i=1}^{3}\right)=$ $\sigma\left(\left(\mathcal{F}^{i}\right)_{i=1}^{3}\right) * \sigma\left(\left(\mathscr{E}^{i}\right)_{i=1}^{3}\right)$.

Let $\left(\mathcal{F}^{i}\right)_{i=1}^{3}$ be a total foliation in $\operatorname{tFol}(A, n)$. Put $\Gamma^{j}=\mathscr{F}^{1} \cap \mathscr{F}^{2}\left(0, Q_{j}\right)$ for $j=0, \ldots, n-1$. For each $k=1,2$ and each $j=0, \ldots, n-1$, there exists a smooth function $\theta_{k}^{j}$ on $\Gamma^{j}$ such that

$$
\cos \left(2 \pi \theta_{k}^{j}(p)\right) e_{x}(p)+\sin \left(2 \pi \theta_{k}^{j}(p)\right) e_{y}(p) \in T \mathcal{F}^{k}(p)
$$

for any $p \in \Gamma^{j}$. We define the rotation $\Theta_{k}\left(\left(\mathcal{F}^{i}\right)_{i=1}^{3}, j\right)$ of $\mathcal{F}^{k}$ along the $j$-th string by

$$
\Theta_{k}\left(\left(\mathcal{F}^{i}\right)_{i=1}^{3}, j\right)=\theta_{k}^{j}\left(1, w_{1}^{j}\right)-\theta_{k}^{j}\left(0, w_{0}^{j}\right),
$$

where $\left\{\left(0, w_{0}^{j}\right),\left(1, w_{1}^{j}\right)\right\}=\partial \Gamma^{j}$. It does not depend on the choice of $\theta_{k}^{j}$. 
For any sufficiently small $\delta>0$ and $j=0, \ldots, n-1$, there exist two maps $f$ and $g$ from $[-2 \delta, 2 \delta]$ to $\mathbb{R}$ such that the holonomy of $\mathcal{F}^{1} \cap \mathcal{F}^{2}$ along $\Gamma^{j}$ is given by the map $\left(0, w_{0}^{j}+(x, y)\right) \mapsto\left(1, w_{1}^{j}+A \cdot(f(x), g(y))\right)$. We define the $\delta$-normalized holonomy of $\mathcal{F}^{1} \cap \mathcal{F}^{2}$ along $j$-th string by the pair $\left(H_{x}^{\delta}\left(\left(\mathcal{F}^{i}\right)_{i=1}^{3}, j\right), H_{y}^{\delta}\left(\left(\mathcal{F}^{i}\right)_{i=1}^{3}, j\right)\right)$ of maps from $[-2,2]$ to $\mathbb{R}$ given by

$$
H_{x}^{\delta}\left(\left(\mathscr{F}^{i}\right)_{i=1}^{3}, j\right)(x)=\delta^{-1} \cdot f(\delta x), \quad H_{y}^{\delta}\left(\left(\mathcal{F}^{i}\right)_{i=1}^{3}, j\right)(y)=\delta^{-1} \cdot g(\delta y) .
$$

We denote by Diff $_{0}([-2,2], 0)$ the set of diffeomorphisms $f$ on $[-2,2]$ such that $f(0)=0$ and $\overline{\{f(x) \neq x\}} \subset]-2,2[$.

Proposition 3.9. For any $A \in \mathrm{SL}(2, \mathbb{Z}), \sigma \in \pi_{0}\left(B_{n}(A)\right), m \in \mathbb{Z}$, and any sequences $\left(f_{j}\right)_{j=0}^{n-1}$ and $\left(g_{j}\right)_{j=0}^{n-1}$ in $\operatorname{Diff}_{0}([-2,2], 0)$, there exists $\left(\mathscr{F}^{i}\right)_{i=1}^{3} \in \mathrm{tFol}(A, n)$ and $\delta>0$ such that

- $\sigma\left(\left(\mathcal{F}^{i}\right)_{i=1}^{3}\right)=\sigma$,

- $\Theta\left(\left(\mathscr{F}^{i}\right)_{i=1}^{3}, j\right)$ does not depend on $j$ and belongs to the interval $[m, m+1[$, and

- $H_{x}^{\delta}\left(\left(\mathscr{F}^{i}\right)_{i=1}^{3}, j\right)=f_{j}$ and $H_{y}^{\delta}\left(\left(\mathscr{F}^{i}\right)_{i=1}^{3}, j\right)=g_{j}$ for any $j=0, \ldots, n-1$.

The rest of the subsection is devoted to the proof of the proposition. We divide it into several lemmas. Put

$$
A_{x y}=\left(\begin{array}{ll}
0 & 1 \\
1 & 0
\end{array}\right), A_{1}=\left(\begin{array}{ll}
1 & 0 \\
1 & 1
\end{array}\right), A_{2}=\left(\begin{array}{ll}
1 & 1 \\
0 & 1
\end{array}\right), A_{*}=\left(\begin{array}{cc}
0 & -1 \\
1 & 1
\end{array}\right) .
$$

They satisfy the following relations:

$$
A_{x y}^{2}=I, A_{x y} \cdot A_{1} \cdot A_{x y}=A_{2}, A_{*}=A_{2}^{-1} \cdot A_{1}, A_{*}^{3}=-I .
$$

Lemma 3.10. The triple $\left(F_{A_{x y}}\left(\mathcal{F}^{2}\right), F_{A_{x y}}\left(\mathscr{F}^{1}\right), F_{A_{x y}}\left(\mathscr{F}^{3}\right)\right)$ is a total foliation in $\operatorname{tFol}\left(A_{x y} \cdot A \cdot A_{x y}\right)$ for any $\left(\mathscr{F}^{i}\right)_{i=1}^{3} \in \operatorname{tFol}(A)$. Moreover, if $\left(\mathscr{F}^{i}\right)_{i=1}^{3} \in \operatorname{tFol}(A, n)$, then the above triple is in $\mathrm{tFol}\left(A_{x y} \cdot A \cdot A_{x y}, n\right)$.

Proof. It is an easy consequence of the identities $F_{A_{x y}}\left(\mathcal{F}_{\text {std }}^{1}\right)=\mathscr{F}_{\text {std }}^{2}$ and $F_{A_{x y}}\left(\mathcal{F}_{\text {std }}^{2}\right)=$ $\mathcal{F}_{\text {std }}^{1}$.

Let $\sigma_{0}$ be the connected component of $B_{n}(I)$ represented by the constant braid $\Gamma_{0}=[0,1] \times\left\{Q_{1}, \ldots, Q_{n-1}\right\}$. The following lemma is an interpretation of the construction in [10] (p. 49-50) in our setting.

Lemma 3.11. For any given $\delta_{0}>0$ and any sequences $\left(f_{j}\right)_{j=0}^{n-1}$ and $\left(g_{j}\right)_{j=0}^{n-1}$ in $\operatorname{Diff}_{0}([-2,2], 0)$ there exist $\left(\mathscr{F}^{i}\right)_{i=1}^{3} \in \operatorname{tFol}(I, n)$ and $\delta \in\left(0, \delta_{0}\right)$ such that $\sigma\left(\left(\mathcal{F}^{i}\right)_{i=1}^{3}\right)=\sigma_{0}, \Theta_{1}\left(\left(\mathscr{F}^{i}\right)_{i=1}^{3}, j\right)=\Theta_{2}\left(\left(\mathscr{F}^{i}\right)_{i=1}^{3}, j\right)=0, H_{x}^{\delta}\left(\left(\mathscr{F}^{i}\right)_{i=1}^{3}, j\right)=$ $f_{j}$, and $H_{y}^{\delta}\left(\left(\mathscr{F}^{i}\right)_{i=1}^{3}, j\right)=g_{j}$ for any $j=0, \ldots, n-1$. 
Proof. Take $\delta \in\left(0, \min \left\{\delta_{0}, 1\right\}\right)$. First, we fix $j_{*} \in\{0, \ldots, n-1\}$ and a diffeomorphism $g \in \operatorname{Diff}_{0}([-2,2], 0)$ and we show the lemma for the case all $f_{j}$ 's and $g_{j}$ 's are the identity except $g_{j_{*}}=g$. Let us modify $\mathcal{F}_{\text {std }}^{1}$ so as to have the holonomy corresponding to $g$. Take a smooth map $\chi_{2}: S^{1} \times[1 / 4,3 / 4] \rightarrow S^{1}$ such that

(1) $\chi_{2}(y, 1 / 4+\epsilon)=y$ and $\chi_{2}(y, 3 / 4-\epsilon)=\chi_{2}(y, 3 / 4)$ for any $y \in[0,1]$ and any small $\epsilon \geq 0$,

(2) $\chi_{2}(y, t)=y$ if $y \notin\left[q_{j_{*}}-2 \delta, q_{j_{*}}+2 \delta\right]$,

(3) $\chi_{2}\left(q_{j_{*}}+y^{\prime}, 3 / 4\right)=q_{j_{*}}+\delta g\left(\delta^{-1} y^{\prime}\right)$ for any $y^{\prime} \in[-\delta, \delta]$,

(4) $\frac{\partial h}{\partial y}(y, t)>0$ and $\left|\frac{\partial h}{\partial t}(y, t)\right|<\eta^{-1}$ for any $(y, t)$.

Remark that $\chi_{2}(\cdot, t)$ is a diffeomorphism of $S^{1}$ for any $t \in[1 / 4,3 / 4]$.

Put $J_{j_{*}}=\left[q_{j_{*}}-(1 / 4 n), q_{j_{*}}+(1 / 4 n)\right] \subset S^{1}$ and $V_{j_{*}}=[1 / 4,3 / 4] \times J_{j_{*}} \times S^{1}$. Since $\partial V_{j_{*}} \cap$ Int $W_{0} \subset\{1 / 4,3 / 4\} \times J_{j_{*}} \times S^{1}$, we can define a foliation $\mathcal{F}_{0}^{1}$ on $W_{0}$ such that $\left.\mathscr{F}_{0}^{1}\right|_{W_{0} \backslash V_{j *}}=\mathscr{F}_{\text {std }}^{1}$ and

$$
\left(\left.\mathcal{F}_{0}{ }^{1}\right|_{j_{*}}\right)(1 / 4, x, y)=\left\{\left(t, x^{\prime}, \chi_{2}(y, t)\right) \mid\left(t, x^{\prime}\right) \in[1 / 4,3 / 4] \times J_{j_{*}}\right\}
$$

for any $(x, y) \in J_{j_{*}} \times[0,1]$. See Figure 7. Since $\left|\frac{\partial h}{\partial t}(y, t)\right|<\eta^{-1}$ for any $(y, t)$, the

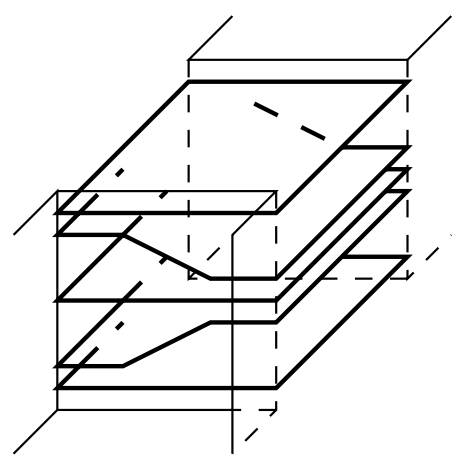

Figure 7. Foliation $\left(\left.\mathcal{F}_{0}^{1}\right|_{V_{*}}\right)$.

foliation $\widetilde{F}_{0}^{1}$ is almost horizontal. By Proposition 3.5, there exists a total foliation $\left(\mathscr{F}^{i}\right)_{i=1}^{3} \in \operatorname{tFol}(I)$ such that $\left.\mathscr{F}^{1}\right|_{W_{0}}=\mathscr{F}_{0}^{1}$ and $\mathscr{F}^{i}=\mathscr{F}_{\text {std }}^{i}$ for $i=2$, 3. Since $\Gamma_{0}$ is tangent to $\mathscr{F}_{0}^{1} \cap \mathscr{F}_{0}^{2},\left(\mathscr{F}^{i}\right)_{i=1}^{3}$ is contained in $\operatorname{tFol}(I, n)$. The holonomy of $\mathcal{F}^{1} \cap \mathscr{F}^{2}$ along the $j_{*}$-th string $\Gamma_{0}^{j_{*}}$ is

$$
\left(0, q_{j_{*}}+x, q_{j_{*}}+y\right) \mapsto\left(1, q_{j_{*}}+x, \chi_{2}\left(q_{j_{*}}+y, 3 / 4\right)\right) \quad \text { for }(x, y) \in[-\delta, \delta]^{2} .
$$

Hence, $H_{x}^{\delta}\left(\left(\mathcal{F}^{i}\right)_{i=1}^{3}, j_{*}\right)$ is the identity map and $H_{y}^{\delta}\left(\left(\mathcal{F}^{i}\right)_{i=1}^{3}, j_{*}\right)=g$. It is easy to see that $H_{x}^{\delta}\left(\left(\mathscr{F}^{i}\right)_{i=1}^{3}, j\right)$ and $H_{y}^{\delta}\left(\left(\mathscr{F}^{i}\right)_{i=1}^{3}, j\right)$ are the identity maps for all $j \neq j_{*}$, and $\Theta_{1}\left(\left(\mathscr{F}^{i}\right)_{i=1}^{3}, j\right)=\Theta_{2}\left(\left(\mathscr{F}^{i}\right)_{i=1}^{3}, j\right)=0$ for any $j=0, \ldots, n-1$. 
By Lemma 3.10, the total foliation $\left(F_{A_{x y}}\left(\mathcal{F}^{2}\right), F_{A_{x y}}\left(\mathscr{F}^{1}\right), F_{A_{x y}}\left(\mathscr{F}^{3}\right)\right)$ is contained in $\operatorname{tFol}(I, n)$. It easy to verify that it satisfies the required conditions for the case $f_{j_{*}}=g$ and all the other $f_{j}$ 's and $g_{j}$ 's are the identity map. Hence, we can obtain the required total foliation for a general sequence $\left(f_{j}, g_{j}\right)_{j=0}^{n-1}$ as a composition of the total foliations given by the above construction.

Lemma 3.12. For any given $\sigma \in \pi_{0}\left(B_{n}(I)\right)$, there exists $\left(\mathscr{F}^{i}\right)_{i=1}^{3} \in \operatorname{tFol}(I, n)$ such that $\sigma\left(\left(\mathscr{F}^{i}\right)_{i=1}^{3}\right)=\sigma$ and $\Theta_{1}\left(\left(\mathscr{F}^{i}\right)_{i=1}^{3}, j\right)=\Theta_{2}\left(\left(\mathscr{F}^{i}\right)_{i=1}^{3}, j\right)=0$ for any $j=0, \ldots, n-1$.

Proof. Fix a smooth function $\alpha$ on [0,1] such that $\alpha(t)=0$ for $t \in[0,1 / 4], \alpha(t)=$ $1 / n$ for $t \in[3 / 4,1]$, and $0 \leq d \alpha / d t(t) \leq \eta^{-1}$ for any $t \in[0,1]$. Put $V_{j}=$ $[0,1] \times\left[q_{j}-(1 / 4 n), q_{j}+(1 / 4 n)\right] \times S^{1}$ and $\Gamma^{j}(y)=\left\{\left(t, q_{j}, y+\alpha(t)\right) \mid t \in[0,1]\right\}$ for $j=0, \ldots, n-1$ and $y \in S^{1}$.

First, for any given $m=0, \ldots, n-1$, there exists $\left(\mathscr{F}_{m}^{i}\right)_{i=1}^{3} \in \operatorname{tFol}(I)$ such that

- $\left.\mathscr{F}_{m}^{1}\right|_{W_{0} \backslash V_{m}}=\left.\mathscr{F}_{\text {std }}^{1}\right|_{W_{0} \backslash V_{m}}, \mathscr{F}_{m}^{2}=\mathscr{F}_{\text {std }}^{2}$, and

- $\Gamma^{m}(y)$ is tangent to $\mathscr{F}_{m}^{1} \cap \widetilde{F}_{m}^{2}$ for any $y \in S^{1}$.

In fact, it can be obtained by the same construction as the total foliation $\left(\mathcal{F}^{i}\right)_{i=1}^{3}$ in the proof of Lemma 3.11 by replacing $\chi_{2}(y, t)$ in the definition of $\mathcal{F}_{0}^{1}$ with $y+\alpha(t)$.

Put $\mathscr{E}_{m}^{1}=F_{A_{x y}}\left(\widetilde{F}_{m}^{2}\right), \mathscr{E}_{m}^{2}=F_{A_{x y}}\left(\widetilde{F}_{m}^{1}\right)$, and $\mathscr{E}_{m}^{3}=F_{A_{x y}}\left(\widetilde{F}_{2}^{3}\right)$. Let $\left(\left(\mathscr{F}_{m}^{i}\right)^{-1}\right)_{i=1}^{3}$ and $\left(\left(\mathscr{C}_{m}^{i}\right)^{-1}\right)_{i=1}^{3}$ be the inverses of $\left(\mathcal{F}_{m}^{i}\right)_{i=1}^{3}$ and $\left(\mathscr{E}_{m}^{i}\right)_{i=1}^{3}$ respectively. Remark that all of them are total foliation in $\mathrm{tFol}(I)$ by Lemma 3.10.

We define $\left(\mathcal{F}_{\sigma_{m}}^{i}\right)_{i=1}^{3} \in \operatorname{tFol}(I)$ by

$$
\mathscr{F}_{\sigma_{m}}^{i}=\mathscr{F}_{m}^{i} *\left(\mathcal{F}_{m+1}^{i}\right)^{-1} * \mathscr{E}_{m+1}^{i} *\left(\mathscr{E}_{m}^{i}\right)^{-1}
$$

and put $\sigma_{m}=\sigma\left(\left(\mathcal{F}_{\sigma_{m}}^{i}\right)_{i=1}^{3}\right)$ for $m=0, \ldots, n-2$. Then, $\left(\mathcal{F}_{\sigma_{m}}^{i}\right)_{i=1}^{3}$ is a total foliation in $\operatorname{tFol}(I, n)$ and $\sigma_{m}$ represents a half twist of $m$-th and $(m+1)$-st strings. See Figure 8.

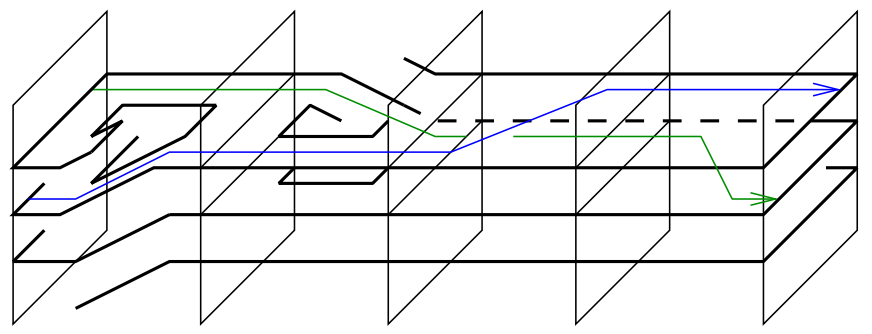

Figure 8. Proof of Lemma 3.12.

Let $\left(\mathcal{F}_{\rho_{m}}^{i}\right)_{i=1}^{3}$ and $\left(\mathcal{F}_{\tau_{m}}^{i}\right)_{i=1}^{3}$ be $n$-times compositions $\left(\mathcal{F}_{m}^{i} * \cdots * \mathcal{F}_{m}^{i}\right)_{i=1}^{3}$ and $\left(\mathscr{E}_{m}^{i} * \cdots * \mathscr{E}_{m}^{i}\right)_{i=1}^{3}$ respectively. Put $\rho_{m}=\sigma\left(\left(\mathcal{F}_{\rho_{m}}^{i}\right)_{i=1}^{3}\right)$ and $\tau_{m}=\sigma\left(\left(\mathcal{F}_{\tau_{m}}^{i}\right)_{i=1}^{3}\right)$. 
We can see that both $\left(\mathcal{F}_{\rho_{m}}^{i}\right)_{i=1}^{3}$ and $\left(\mathcal{F}_{\tau_{m}}^{i}\right)_{i=1}^{3}$ are total foliations in $\operatorname{tFol}(I, n)$ and $\rho_{m}$ (resp. $\left.\tau_{m}\right)$ is represented by a braid such that the $m$-th string winds once in the $y$-(resp. $x$-)direction and other strings are fixed.

It is easy to see that $\Theta_{k}\left(\left(\mathcal{F}_{\sigma}^{i}\right)_{i=1}^{3}, j\right)=0$ for any $k=1,2, m=0, \ldots, n-1$, and $\sigma=\sigma_{m}, \rho_{m}, \tau_{m}$. It is known that $\left\{\sigma_{m}, \rho_{m}, \tau_{m} \mid m=0, \ldots, n-1\right\}$ generates $\pi_{0}\left(B_{n}(I)\right)$ (see e.g. [1] or [9]). Hence, we can obtain the required total foliation as a composition of the total foliations constructed above and their inverses.

Lemma 3.13. There exists $\left(\mathcal{F}_{1}^{i}\right)_{i=1}^{3} \in \operatorname{tFol}\left(A_{1}, n\right)$ satisfying

$$
\Theta_{1}\left(\left(\mathscr{F}_{1}^{i}\right)_{i=1}^{3}, j\right)=1 / 8, \quad \Theta_{2}\left(\left(\mathscr{F}_{1}^{i}\right)_{i=1}^{3}, j\right)=0
$$

for any $j=0, \ldots, n-1$.

Proof. Take a smooth map $\bar{\chi}_{3}:[0,1] \rightarrow \mathbb{R}$ such that

- $0 \leq \frac{d \bar{\chi}_{3}}{d x}(x) \leq \eta^{-1}$ holds for any $x \in[0,1]$,

- $\bar{\chi}_{3}(x)=0$ holds for any $x \in[0,1-(9 / 16 n)]$, and $\bar{\chi}_{3}(x)=1$ holds for any $x \in[1-(7 / 16 n), 1]$.

It induces a map $\chi_{3}: S^{1} \rightarrow S^{1}$ of degree 1 . We define a diffeomorphism $G$ of $W_{0}$ by $G(t, x, y)=\left(t, x, y+\chi_{3}(x)\right)$ if $t \in[3 / 4,1]$ and $G(t, x, y)=(t, x, y)$ otherwise. It is well-defined and satisfies $G\left(\widetilde{F}_{\text {std }}^{i} \mid W_{0}\right)=\mathscr{F}_{\text {std }}^{i} \mid W_{0}$ for $i=2,3$. Since $G\left(\mathcal{F}_{\text {std }}^{1} \mid W_{0}\right)$ is almost horizontal, Proposition 3.5 implies that there exists an extension $\mathcal{E}$ of $G\left(\mathcal{F}_{\text {std }}^{1} \mid W_{0}\right)$ to $W$ which is transverse to $\mathcal{F}_{\text {std }}^{2}$ and $\mathcal{F}_{\text {std }}^{3}$. Remark that the constant braid $\Gamma_{0}$ is tangent to $\mathscr{E} \cap \mathcal{F}_{\text {std }}^{2}$.

Since $\chi_{3}(x)-x$ is a map of degree 0 , we can take a smooth function $\alpha$ on $S^{1} \times[0,1]$ such that $\alpha(x, t)=0$ for $(x, t) \in S^{1} \times[0,3 / 4]$ and $x=\chi_{3}(x)+\alpha(x, t)$ for $(x, t) \in S^{1} \times[7 / 8,1]$. We define a diffeomorphism $\bar{G}$ of $W$ by $\bar{G}(t, x, y)=$ $(t, x, y+\alpha(x, t))$. Remark that $\bar{G} \circ G(t, x, y)=(t, x, y)$ if $t \in[0,3 / 4]$ and $\bar{G} \circ G(t, x, y)=\left(t, A_{1}(x, y)\right)$ if $t \in[7 / 8,1]$. Put $\mathscr{F}_{1}^{1}=\bar{G}(\mathscr{G}), \mathcal{F}_{1}^{i}=\bar{G}\left(\mathscr{F}_{\text {std }}^{i}\right)$ for $i=2,3$ and $\Gamma=\bar{G}\left(\Gamma_{0}\right)$. Then, $\left(\mathcal{F}_{1}^{i}\right)_{i=1}^{3}$ is a total foliation contained in $\operatorname{tFol}\left(A_{1}\right)$ and $\bar{G}\left(\Gamma_{0}\right)$ is a braid in $B_{n}\left(A_{1}\right)$ which is tangent to $\mathscr{F}_{1}^{1} \cap \mathcal{F}_{1}^{2}$. Therefore, $\left(\mathcal{F}_{1}^{i}\right)_{i=1}^{3}$ an element of $\operatorname{tFol}\left(A_{1}, n\right)$. See Figure 9. Since $\mathcal{F}_{1}{ }_{1}$ is almost horizontal on $W_{0}$, we have $\Theta_{1}\left(\left(\mathscr{F}_{1}^{i}\right)_{i=1}^{3}, j\right)=1 / 8$ for any $j=0, \ldots, n-1$. By the transversality of $\mathscr{F}_{1}{ }_{1}$ and $\mathscr{F}_{1}^{2}$, we also have $\left.\Theta_{2}\left(\left(\mathcal{F}_{1}^{i}\right)_{i=1}^{3}, j\right)-\Theta_{1}\left(\left(\mathcal{F}_{1}^{i}\right)_{i=1}^{3}, j\right) \in\right]-1 / 2,1 / 2[$. It implies $\Theta_{2}\left(\left(\mathcal{F}_{1}^{i}\right)_{i=1}^{3}, j\right)=0$ for any $j=0, \ldots, n-1$.

Starting from the total foliation $\left(\mathscr{F}_{1}^{i}\right)_{i=1}^{3}$ in Lemma 3.13, we define a total foliation $\left(\mathcal{F}_{2}^{i}\right)_{i=1}^{3}$ by $\mathscr{F}_{2}^{1}=F_{A_{x y}}\left(\mathcal{F}_{1}^{2}\right), \mathscr{F}_{2}^{2}=F_{A_{x y}}\left(\mathscr{F}_{1}^{1}\right)$ and $\mathscr{F}_{2}^{3}=F_{A_{x y}}\left(\mathcal{F}_{1}^{3}\right)$. By Lemma 3.10 and the third equation of (6), we have $\left(\mathscr{F}_{2}^{i}\right)_{i=1}^{3} \in \operatorname{tFol}\left(A_{2}\right)$. By (7), we also have

$$
\Theta_{1}\left(\left(\mathcal{F}_{2}^{i}\right)_{i=1}^{3}, j\right)=0, \quad \Theta_{2}\left(\left(\widetilde{F}_{2}^{i}\right)_{i=1}^{3}, j\right)=-1 / 8 .
$$

for any $j=0, \ldots, n-1$. 

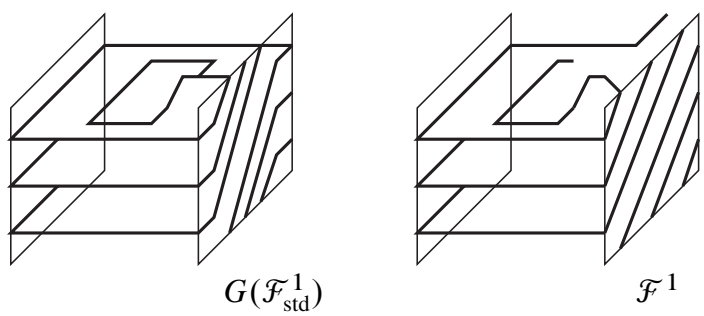

Figure 9. Proof of Lemma 3.13.

Lemma 3.14. For any $m \in \mathbb{Z}$, there exists $\left(\mathcal{F}^{i}\right)_{i=1}^{3} \in \mathrm{t} \operatorname{Fol}(I, n)$ such that

$$
\Theta_{1}\left(\left(\mathcal{F}^{i}\right)_{i=1}^{3}, j\right)=\Theta_{2}\left(\left(\mathcal{F}^{i}\right)_{i=1}^{3}, j\right)=m
$$

for any $j=0, \ldots, n-1$.

Proof. Let $\left(\left(\mathcal{F}_{2}^{i}\right)^{-1}\right)_{i=1}^{3}$ be the inverse of $\left(\mathcal{F}_{2}^{i}\right)_{i=1}^{3}$. Put $\mathscr{g}^{i}=\mathscr{F}_{1}^{i} *\left(\mathcal{F}_{2}^{i}\right)^{-1}$ for $i=1,2,3$. Since $A_{*}=A_{2}^{-1} \cdot A_{1}$, we have $\left(\mathscr{G}^{i}\right)_{i=1}^{3} \in \operatorname{tFol}\left(A_{*}, n\right)$. The equations (7) and (8), we also have $\Theta_{1}\left(\left(\mathscr{G}^{i}\right)_{i=1}^{3}, j\right)=1 / 4$ and $\Theta_{2}\left(\left(\mathscr{G}^{i}\right)_{i=1}^{3}, j\right)=1 / 8$ for any $j=0, \ldots, n-1$. Let $\left(\mathscr{G}_{k}^{i}\right)_{i=1}^{3}$ be the $k$-times composition of $\left(\mathscr{G}^{i}\right)_{i=1}^{3}$. Since $A_{*}^{3}=-I$, we have $\Theta_{1}\left(\left(\mathscr{G}_{3}^{i}\right)_{i=1}^{3}, j\right)=1 / 2$ and $\Theta_{2}\left(\left(\mathscr{G}_{3}^{i}\right)_{i=1}^{3}, j\right)=1 / 2$ for any $j=0, \ldots, n-1$. Hence, $(6 m)$-times composition $\left(\mathscr{E}_{6 m}^{i}\right)_{i=1}^{3}$ of $\left(\mathscr{E}^{i}\right)_{i=1}^{3}$ is the required total foliation for $m \geq 0$. For $m<0$, it is sufficient to take the inverse $\left(\left(\mathscr{E}_{|6 m|}^{i}\right)^{-1}\right)_{i=1}^{3}$ of $\left(\mathscr{E}_{|6 m|}^{i}\right)_{i=1}^{3}$.

Lemma 3.15. For any $A \in \operatorname{SL}(2, \mathbb{Z})$, there exist $\left(\mathcal{F}^{i}\right)_{i=1}^{3} \in \operatorname{tFol}(A, n)$ such that $\Theta_{1}\left(\left(\mathcal{F}^{i}\right)_{i=1}^{3}, j\right)$ and $\Theta_{2}\left(\left(\mathcal{F}^{i}\right)_{i=1}^{3}, j\right)$ does not depends on $j$.

Proof. It is an immediate consequence of Lemma 3.13 and the fact that $\left\{A_{1}, A_{2}\right\}$ generates $\operatorname{SL}(2, \mathbb{Z})$.

Finally, Proposition 3.9 is an immediate consequence of Lemmas 3.11, 3.12, 3.14, and 3.15 .

3.3. Braided knots in embedded solid tori. Let $\psi$ be an embedding from $Z=$ $S^{1} \times D^{2}$ to an oriented three-dimensional manifold $M$. We say $\psi$ is a 0 -framed null-homotopic embedding if $K_{0}=\psi\left(S^{1} \times\{(0,0)\}\right)$ is null-homotopic and $\psi$ is a 0 -framed tubular coordinate of $K_{0}$. We also say $\psi$ is unknotted if $K_{0}$ is unknotted.

We say a smooth link $L$ in $M$ is $\psi$-braided if $L \subset \psi(Z)$ and $\psi^{-1}(L)$ is transverse to the production foliation $\left\{t \times D^{2}\right\}_{t \in S^{1}}$. Remark that any component of $L$ is a $\psi$ braided knot. 
Definition 3.16. Let $L$ be a $\psi$-braided oriented knot or link. We denote by $n(L ; \psi)$ the cardinality of $\psi^{-1}(L) \cap\left(0 \times D^{2}\right)$. We define the $(\psi, n)$-framing of $L$ in $M$ by a vector field

$$
v_{n}(\psi(t, w))=D \psi\left(\cos (2 \pi n t) e_{x}(t, w)+\sin (2 \pi n t) e_{y}(t, w)\right)
$$

for $(t, w) \in \psi^{-1}(L)$, where $\left(e_{t}, e_{x}, e_{y}\right)$ is the standard frame on $S^{1} \times D^{2} \subset S^{1} \times \mathbb{R}^{2}$.

Remark that $(\psi, n)$-framing of $K$ may not be the $n$-framing (in the sense of Definition 2.12) even if $\psi$ is 0 -framed and unknotted. See Lemma 3.18.

Let $\psi_{0}$ be a 0 -framed unknotted embedding of $Z$ into $\mathbb{R}^{3}$ defined by $\psi_{0}(t, x, y)=$ $((x+2) \cos 2 \pi t,(x+2) \sin 2 \pi t, y)$ and $P_{x y}$ denote the projection from $\mathbb{R}^{3}$ to $\mathbb{R}^{2}$ given by $P_{x y}(x, y, z)=(x, y)$. For any given 0 -framed unknotted embedding $\psi$ of $Z$ into $M$, we can take an embedding $\varphi$ of $\mathbb{R}^{3}$ into $M$ so that $\varphi^{-1} \circ \psi=\psi_{0}$. Take a $\psi$-braided link $L$ in $M$. The map $\varphi$ can be perturbed into another embedding $\varphi_{1}$ such that the map $P_{x y} \circ \varphi_{1}$ is a regular projection associated with $\varphi_{1}^{-1}(L)$. See $e$.g. [14] for the definition of a regular projection and a link diagram. For any component $K$ of $L$, let $\omega_{ \pm}(K ; \psi)$ be the number of positive and negative crossings in the diagram $P_{x y} \circ \varphi_{1}^{-1}(K)$. See Figure 10 . We put $\omega(K ; \psi)=\omega_{+}(K ; \psi)-\omega_{-}(K ; \psi)$. Remark

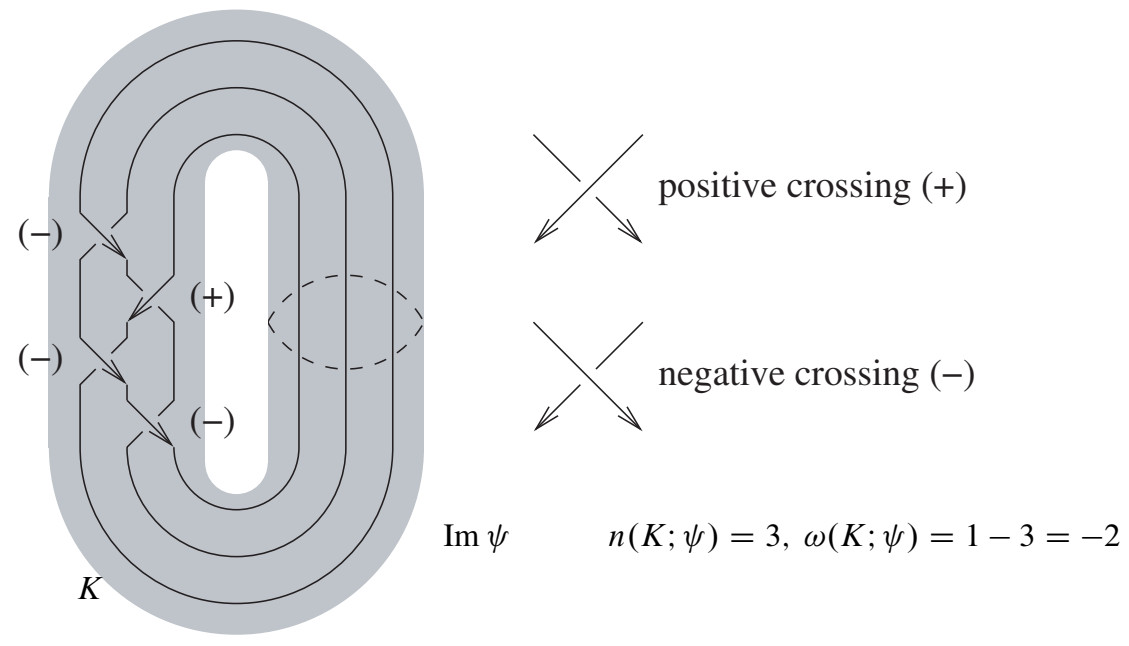

Figure 10. A link diagram of a braided knot.

that $\omega(K ; \psi)$ and $n(K ; \psi)$ depend only on the isotopy class of $K$ as a $\psi$-braided knot.

We show two lemmas, which give relations between $n(K ; \psi), \omega(K ; \psi)$ and the framing of $K$. 
Lemma 3.17. Let $\psi$ be a 0 -framed unknotted embedding from $Z$ to $M$ and $K$ be a $\psi$-braided knot in $M$. Then, $\omega(K ; \psi)+n(K ; \psi)$ is odd.

Proof. Since $K$ is connected, it induces a cyclic permutation on the $n(K ; \psi)$-points set $\psi^{-1}(K) \cap\left(0 \times D^{2}\right)$. Then, the signature of the permutation is $(-1)^{n(K ; \psi)+1}$. Since the induced permutation is the product of $\left(\omega_{+}(K ; \psi)+\omega_{-}(K ; \psi)\right)$ transpositions, its signature is also $(-1)^{\left(\omega_{+}(K ; \psi)+\omega_{-}(K ; \psi)\right)}$. In particular, $n(K ; \psi)-\left(\omega_{+}(K ; \psi)+\right.$ $\left.\omega_{-}(K ; \psi)\right)$ is odd. Hence, also $\omega(K ; \psi)+n(K ; \psi)$ is.

Lemma 3.18. Let $\psi$ be a 0 -framed unknotted embedding from $Z$ to $M$ and let $K$ be a $\psi$-braided knot in $M$. Then, the $(\psi, m)$-framing of $K$ coincides with the $(\omega(K ; \psi)+m \cdot n(K ; \psi))$-framing of $K$ as a null-homotopic knot in $M$.

Proof. Suppose that the $(\psi, 0)$-framing of $K$ is the $n_{0}$-framing. It is easy to see that the $(\psi, m)$-framing is $n_{0}+m \cdot n(K ; \psi)$. Under the identification of $\psi(Z)$ and the standard torus $\psi_{0}(Z)$, the $(\psi, 0)$-framing gives the blackboard framing, that is, the one transverse to the projection to the link diagram. By a well-known result in knot theory (see e.g. [8], Proposition 4.5.8), it coincides with the $\omega(K ; \psi)$-framing of $K$. Hence, we have $n_{0}=\omega(K ; \psi)$.

3.4. The trefoil complement. In this subsection, we construct a total foliation on $S^{3}$ containing $\mathcal{R}$-components such that their cores form an arbitrary given link. It will be done by using the fibration of the complement of the trefoil. Note that the same construction can be done for other fibered knot with one-punctured torus fibers, e.g. the figure-eight knot.

Let $A_{*}$ be the matrix defined in (5) and $M_{*}$ be the mapping torus $W /(0, w) \sim$ $\left(1, A_{*} \cdot w\right)$ of the linear map defined by $A_{*}$. By $P_{M_{*}}$, we denote the natural projection from $W$ to $M_{*}$. Since any total foliation $\left(\mathscr{F}^{i}\right)_{i=1}^{3} \in \operatorname{tFol}\left(A_{*}\right)$ is compatible with the projection $P_{M_{*}}$ at $\partial W$, we can define a total foliation $\left(P_{M_{*}}\left(\mathcal{F}^{i}\right)\right)_{i=1}^{3}$ on $M_{*}$ such that $P_{M_{*}}\left(\mathscr{F}^{i}\right)\left(P_{M_{*}}(t, w)\right)=P_{M_{*}}\left(\mathscr{F}^{i}(t, w)\right)$ for any $i=1,2,3$ and $(t, w) \in W$.

Since $Q_{0}=(0,0)+\mathbb{Z}^{2}$ is a fixed point of $A_{*}, P_{M_{*}}\left([0,1] \times Q_{0}\right)$ is a knot in $M_{*}$. We denote it by $K_{0}$. Fix an embedding $\psi_{K_{0}}: Z \rightarrow M_{*}$ such that $\psi_{K_{0}}\left(S^{1} \times\{(0,0)\}\right)=$ $K_{0}$ and $\psi_{K_{0}}\left(t \times D^{2}\right) \subset P_{M_{*}}\left(t \times D^{2}(1 / 8 n)\right)$.

Proposition 3.19. For any $\psi_{K_{0}}$-braided link $L$ and $m \in \mathbb{Z}$, there exists a total foliation $\left(\mathcal{F}^{i}\right)_{i=1}^{3}$ on $M_{*}$ such that each component of $L$ is the core of an $\mathcal{R}$-component and its framing determined by $\mathscr{F}^{1}$ is the $\left(\psi_{K_{0}}, m\right)$-framing.

Proof. We take a smooth function $\theta$ on $[0,1]$ such that $D \psi\left(e_{x}(t, 0,0)\right)$ is parallel to $D P_{M_{*}}\left(\cos \theta(t) e_{x}(t, 0,0)+\sin \theta(t) e_{y}(t, 0,0)\right)$. For $m \in \mathbb{Z}$, we define a vector field $\bar{v}_{m}$ on $W$ by

$$
\bar{v}_{m}(t, w)=\cos (2 \pi m t+\theta(t)) e_{x}(t, w)+\sin (2 \pi m t+\theta(t)) e_{y}(t, w)
$$


for $(t, w) \in W$. Then, the vector field $v_{m}=D P_{M_{*}}\left(\bar{v}_{m}\right)$ on $M_{*}$ is well-defined and the restriction of $v_{m}$ to a knot $K$ in $M_{*}$ gives the $\left(\psi_{K_{0}}, m\right)$-framing of $K$.

Now let $\left\{F_{s}\right\}_{s \in[0,1]}$ be an isotopy of $M_{*}$ so that $F_{0}$ is the identity map, $F_{1}(L) \in$ $B_{n}\left(A_{*}\right)$ for some $n \geq 1$, and $F_{S}\left(P_{M_{*}}\left(t \times \mathbb{T}^{2}\right)\right)=P_{M_{*}}\left(t \times \mathbb{T}^{2}\right)$ for any $s \in[0,1]$ and $t \in S^{1}$. Put $L^{\prime}=F_{1}(L)$. Since $\left\{\left(F_{S}(L), v_{m}\right)\right\}_{s \in[0,1]}$ is an isotopy between framed knots $\left(L, v_{m}\right)$ and $\left(L^{\prime}, v_{m}\right)$, it is sufficient to find a total foliation $\left(\mathcal{F}^{i}\right)_{i=1}^{3}$ such that each component of $L^{\prime}$ is the core of an $\mathcal{R}$-component and its framing determined by $\mathcal{F}^{1}$ coincides with the one represented by $v_{m}$.

Take a subset $S_{L}$ of $\{0, \ldots, n-1\}$ such that each component of $L^{\prime}$ contains exactly one point of $\left\{P_{M_{*}}\left(0, Q_{j}\right) \mid j \in S_{L}\right\}$. By $H_{\mathcal{R}}: \mathbb{R} \rightarrow \mathbb{R}$, we denote the holonomy map of the foliation $\widehat{\mathcal{R}}^{1}$ (see Subsection 2.2 for the definition of $\widehat{\mathcal{R}}^{1}$ ). Proposition 3.9 implies that there exist $n \geq 1, \delta>0$, and $\left(\mathscr{E}^{i}\right)_{i=1}^{3} \in \operatorname{tFol}\left(A_{*}, n\right)$ which satisfy the following properties:

- $P_{M_{*}}^{-1}\left(L^{\prime}\right)$ is tangent to $\mathscr{G}^{1} \cap \mathscr{\mathcal { E }}^{2}$,

- $\Theta_{1}\left(\left(\mathscr{E}^{i}\right)_{i=1}^{3}, j\right)=m+\theta(1)$ for any $j=0, \ldots, n-1$, and

- both $H_{x}^{\delta}\left(\left(\mathscr{G}^{i}\right)_{i=1}^{3}, j\right)$ and $H_{y}^{\delta}\left(\left(\mathscr{g}^{i}\right)_{i=1}^{3}, j\right)$ are conjugate to $\left.H_{\mathcal{R}}\right|_{[-2,2]}$ for $j \in$ $S_{L}$ and the identity map otherwise.

The total foliation $\left(\mathscr{E}^{i}\right)_{i=1}^{3}$ induces a total foliation $\left(\mathcal{F}_{*}^{i}=P_{M_{*}}\left(\mathscr{E}^{i}\right)\right)_{i=1}^{3}$ on $M_{*}$. For each component $K^{\prime}$ of $L^{\prime}$, the first and the last condition above imply that there exists an embedding $\psi_{K^{\prime}}: Z \rightarrow M_{*}$ such that $\psi_{K^{\prime}}\left(S^{1} \times\{(0,0)\}\right)=K^{\prime}$ and $\psi_{K^{\prime}}\left(\mathcal{R}^{i}\right)=$

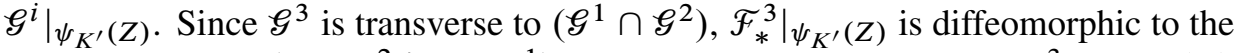
product foliation $\left\{t \times D^{2} \mid t \in S^{1}\right\}$. Hence, a turbularization of $\widetilde{F}_{*}^{3}$ at $\psi_{K^{\prime}}(Z)$ produces an $\mathcal{R}$-component whose core is $K^{\prime}$.

By the second condition in the above, the framing on $L^{\prime}$ determined by $\mathcal{F}_{*}^{1}$ coincides with the one represented by $v_{m}$. Hence, we can obtain the required total foliation by a turbularization along a tubular neighborhood of $L^{\prime}$.

Let $K^{3}$ be the right-handed trefoil on $S^{3}$. It is known that $K^{3}$ is a fibered knot with monodromy matrix $A_{*}$ (see e.g. Section 10.I in [14]). Hence, there exists a diffeomorphism $\varphi$ from $M_{*} \backslash K_{0}$ to $S^{3} \backslash K^{3}$, an embedding $\psi_{K^{3}}$ from $Z$ to $S^{3}$, and an integer $m_{*}$ such that $\psi_{K^{3}}\left(S^{1} \times\{(0,0)\}\right)=K^{3}$ and

$$
\left.\varphi \circ \psi_{K_{0}}(t, r \cos (2 \pi \theta), r \sin (2 \pi \theta))\right)=\psi_{K^{3}}\left(\theta+m_{*} t, r \cos (2 \pi t), r \sin (2 \pi t)\right)
$$

for any $t, \theta \in S^{1}$ and $r \in[0,1]$. Note that $\psi_{K^{3}}$ is a 0 -framed null-homotopic embedding as $\psi_{K^{3}}\left(S^{1} \times\{(1,0)\}\right)$ is contained in a Seifert surface $\overline{\varphi\left(P_{M_{*}}\left(0 \times\left\{\mathbb{T}^{2} \backslash Q_{0}\right\}\right)\right.}$ of $K^{3}$. We define another embedding $\psi_{0}$ from $Z$ to $S^{3}$ by

$$
\psi_{0}(t, x, y)=\psi_{K^{3}}\left(\frac{x}{4}, \frac{y+2}{4} \cos (2 \pi t), \frac{y+2}{4} \sin (2 \pi t)\right) .
$$


Then, the core $\psi_{0}\left(S^{1} \times\{(0,0)\}\right)$ bounds a disk $D_{0}=\psi_{K^{3}}\left(0 \times D^{2}(1 / 2)\right\}$. In particular, $\psi_{0}\left(S^{1} \times\{(0,0)\}\right)$ is a meridian of $K^{3}$. Since $\psi_{0}\left(S^{1} \times\{(0,-1)\}\right)$ is contained in $D_{0}, \psi_{0}$ is a 0 -framed unknotted embedding. See Figure 11.

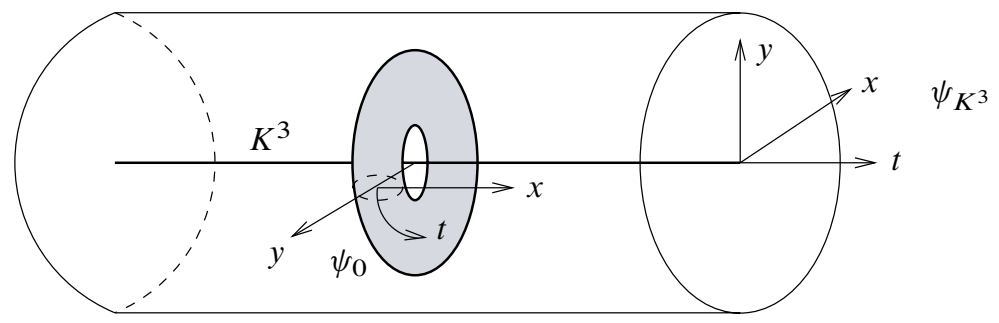

Figure 11. The 0 -framed unknotted embedding $\psi_{0}$.

Lemma 3.20. For any $\psi_{0}$-braided link $L, L^{\prime}=\varphi^{-1}(L)$ is $\psi_{K_{0}}$-braided and $\varphi$ maps the $\left(\psi_{0}, n-m_{*}\right)$-framing of $L^{\prime}$ to the $\left(\psi_{K_{0}}, n\right)$-framing of $L$.

Proof. By direct calculation, we have

$$
\psi_{0}(t, x, y)=\varphi \circ \psi_{K_{0}}\left(t, \frac{y+2}{4} \cos 2 \pi\left(\frac{x}{4}-m_{*} t\right), \frac{y+2}{4} \sin 2 \pi\left(\frac{x}{4}-m_{*} t\right)\right)
$$

for $(t, x, y) \in Z$. It implies $\varphi^{-1} \circ \psi_{0}\left(t \times D^{2}\right) \subset \psi_{K_{0}}\left(t \times D^{2}\right)$ for any $t \in S^{1}$. Hence, $L^{\prime}=\varphi^{-1}(L)$ is $\psi_{K_{0}}$-braided for any $\psi_{0}$-braided link $L$. The above equation also implies that $\varphi^{-1}$ maps the $\left(\psi_{0}, 0\right)$-framing of $L$ to the $\left(\psi_{K_{0}},-m_{*}\right)$-framing of $L^{\prime}$. Since the map $\psi_{K_{0}}^{-1} \circ \varphi^{-1} \circ \psi_{0}$ preserves the orientation of $t \times D^{2}$, it implies that the $\varphi^{-1}$ maps the $\left(\psi_{0}, n\right)$-framing of $L$ to the $\left(\psi_{K_{0}}, n-m_{*}\right)$-framing of $L^{\prime}$.

Proposition 3.21. For any $\psi_{0}$-braided link $L$, there exists a total foliation $\left(\mathcal{F}^{i}\right)_{i=1}^{3}$ on $S^{3}$ such that any connected component $K$ of $L$ is the core of an $\left(\omega\left(K ; \psi_{0}\right)+\right.$ $\left.n\left(K ; \psi_{0}\right)\right)$-framed $\mathcal{R}$-component.

Proof. The link $L_{*}=K_{0} \cup \varphi^{-1}(L)$ in $M_{*}$ is $\psi_{K_{0}}$-braided. By Proposition 3.19, there exists a total foliation $\left(\mathscr{F}_{*}^{i}\right)_{i=1}^{3}$ on $M_{*}$ such that each component of $L_{*}$ is the core of an $\mathcal{R}$-component and the framing determined by $\mathcal{F}_{*}{ }^{1}$ coincides with the $\left(\psi_{K_{0}}, 1-m_{*}\right)$-framing. Let $R_{0}$ be the $\mathcal{R}$ component of $\left(\mathcal{F}_{*}^{i}\right)_{i=1}^{3}$ whose core is $K_{0}$. Without loss of generality, we may assume that $R_{0}=\psi_{K_{0}}\left(S^{1} \times D^{2}(\epsilon)\right)$ for some $\epsilon>0$. Then, $a_{\mathcal{R}}\left(R_{0}\right)$ is represented by a curve

$$
C_{0}=\psi_{K_{0}}\left(\left\{\left(t, \epsilon \cos \left(2 \pi\left(1-m_{*}\right) t\right), \epsilon \sin \left(2 \pi\left(1-m_{*}\right) t\right)\right) \mid t \in S^{1}\right\}\right)
$$

with a suitable orientation. 
Put $\mathscr{F}^{i}=\varphi\left(\left.\mathscr{F}_{*}^{i}\right|_{\bar{M}_{*} \backslash R}\right)$ for $i=1,2,3$ and $R_{0}^{\prime}=\psi_{K^{3}}\left(S^{1} \times D^{2}(\epsilon)\right)$. Then, $\left(\mathcal{F}^{i}\right)_{i=1}^{3}$ is a total foliation on $\overline{S^{3} \backslash R_{0}^{\prime}}$ with an $\mathcal{R}$-boundary $\partial R_{0}^{\prime}$. By (9), we have

$$
\varphi\left(C_{0}\right)=\psi_{K^{3}}(\{(t, \epsilon \cos (2 \pi t), \epsilon \sin (2 \pi t))\}) .
$$

Since $\psi_{K^{3}}$ is a 0 -framed embedding, we can extend $\left(\mathscr{F}^{i}\right)_{i=1}^{3}$ so that $R_{0}^{\prime}$ is a $(+1)$ framed $\mathcal{R}$-component with $C\left(R_{0}\right)=K^{3}$. Since the framing on $L_{*}$ determined by $\mathscr{F}_{*}{ }^{1}$ coincides with the $\left(\psi_{K_{0}}, 1-m_{*}\right)$-framing, Lemma 3.20 implies that the framing on $L$ determined by $\mathcal{F}^{1}$ is the $\left(\psi_{0}, 1\right)$-framing. Since $\psi_{0}$ is a 0 -framed unknotted embedding, it gives the $\left(\omega\left(K ; \psi_{0}\right)+n\left(K ; \psi_{0}\right)\right)$-framing on each component $K$ of $L$ by Lemma 3.18. In particular, each component $K$ of $L$ is the core of an $\left(\omega\left(K ; \psi_{0}\right)+\right.$ $\left.n\left(K ; \psi_{0}\right)\right)$-framed $\mathcal{R}$-component.

3.5. A proof of Hardorp's theorem. First, we show that we can change the framing of an $\mathcal{R}$-component by an arbitrary even integer.

Lemma 3.22. Suppose that a total foliation $\left(\mathcal{F}^{i}\right)_{i=1}^{3}$ on $S^{3}$ admits a $k$-framed $\mathcal{R}$ component $R$. Then, for any integer $n$, there exists a total foliation $\left(\mathscr{F}_{n}^{i}\right)_{i=1}^{3}$ on $S^{3}$ such that it admits a $(k+2 n)$-framed $\mathcal{R}$-component $R^{\prime}$ with $C\left(R^{\prime}\right)=C(R)$ and $\left.\mathscr{F}_{n}^{i}\right|_{S^{3} \backslash R}=\left.\mathscr{F}^{i}\right|_{S^{3} \backslash R}$ for $i=1,2,3$.

Proof. Let $\psi$ be a 0 -framed unknotted embedding of $Z$ into $S^{3}$ and $L=K_{1} \cup K_{2}$ be the $\psi$-braided link in Figure 12. By Proposition 3.21, we can take a total foliation $\left(\mathscr{G}^{i}\right)_{i=1}^{3}$ on $S^{3}$ which admits $\mathcal{R}$-components $R_{1}$ and $R_{2}$ such that $K_{i}$ is the core of

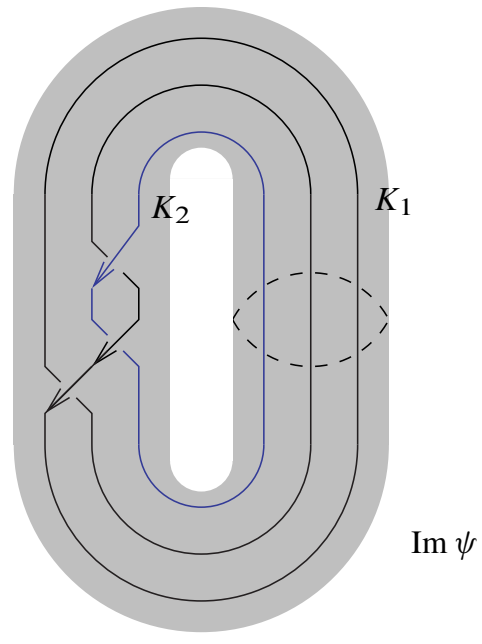

Figure 12. The link $L$ for the proof of Lemma 3.22. 
$R_{i}$ for $i=1,2$ and the framings of $R_{1}$ and $R_{2}$ are +3 and +1 , respectively. Put $M_{+}=\overline{S^{3} \backslash R_{2}}$. Since $L$ is a positive Hopf link, $M_{+}$is diffeomorphic to $Z$. Hence, there exists a diffeomorphism $\varphi_{+}: Z \rightarrow M_{+}$such that $K_{1}=\varphi_{+}\left(S^{1} \times\{(0,0)\}\right)$ coincides with the core of $R_{1}$ as oriented knots and $\left(\left.\varphi_{+}\right|_{\partial Z}\right)_{*}\left(a_{t}\right)=a_{\mathcal{R}}\left(\partial R_{2}\right)$, where $a_{t}$ is the homology class in $H_{1}(\partial Z, \mathbb{Z})$ represented by a map $t \mapsto(t, 1,0)$. It is easy to see that $K_{1}$ is a $\varphi_{+}$-braided knot. Since $K_{1}$ is $(+3)$-framed and $K_{2}$ is $(+1)$-framed, $\mathscr{E}_{*}^{1}$ gives the $\left(\varphi_{+}, 2\right)$-framing of $K_{1}$.

The lemma for $n=0$ is trivial. First, we show the lemma for $n=1$. Let $\psi: Z \rightarrow$ $R$ be a diffeomorphism such that $\psi\left(S^{1} \times\{(0,0)\}\right)=C(R)$ as oriented knots and $\psi_{*}\left(a_{t}\right)=a_{\mathcal{R}}(\partial R)$. By Proposition 2.11, if we choose $\varphi_{+}$suitably in its isotopy class, then we can obtain a total foliation $\left(\mathscr{F}_{1}^{i}\right)_{i=1}^{3}$ on $S^{3}$ such that $\left.\mathscr{F}_{1}^{i}\right|_{R}=\psi \circ \varphi_{+}^{-1}\left(\left.\mathscr{E}_{*}^{i}\right|_{M_{+}}\right)$ and $\left.\mathscr{F}_{1}^{i}\right|_{S^{3} \backslash R}=\left.\mathscr{F}^{i}\right|_{\overline{S^{3} \backslash R}}$ for $i=1,2,3$. Since $\psi$ is a $k$-framed embedding and $\mathscr{E}_{*}^{1}$ gives the $\left(\varphi_{+}, 2\right)$-framing of $C\left(R_{1}\right)=K_{1}, \psi \circ \varphi_{+}^{-1}\left(R_{1}\right)$ is a $(k+2)$-framed $\mathcal{R}$ component with $C\left(\psi \circ \varphi_{+}^{-1}\left(R_{1}\right)\right)=C(R)$. By inductive construction, it gives the proof for $n \geq 1$.

Using the fact that $M_{-}=\overline{S^{3} \backslash R_{1}}$ is diffeomorphic to $Z$, we can take a diffeomorphism $\varphi_{-}: M_{+} \rightarrow Z$ such that $\varphi_{-}\left(K_{2}\right)=S^{1} \times\{(0,0)\}$ as oriented knots and $\left(\varphi_{-}\right)_{*}\left(a_{\mathcal{R}}\left(\partial R_{2}\right)\right)=a_{t}$. Similar to $\varphi_{+}, K_{2}$ is $\psi_{-}$-braided and $\mathscr{G}_{*}^{1}$ gives the $\left(\varphi_{-},-2\right)$ framing of $K_{2}$. Hence, the same construction to the above completes the proof for $n \leq-1$.

Now, we give an alternative proof of Hardorp's theorem [10] with some extension.

Theorem 3.23. For any given closed three-dimensional manifold $M$ equipped with a spin structure $s$, there exists a total foliation $\left(\mathscr{F}^{i}\right)_{i=1}^{3}$ such that

- $s$ is the spin structure given by $\left(\mathcal{F}^{i}\right)_{i=1}^{3}$,

- $\left(\mathscr{F}^{i}\right)_{i=1}^{3}$ admits two unknotted $\mathcal{R}$-components $R_{+}$and $R_{-}$,

- $R_{+}$is (+1)-framed and $R_{-}$is (-1)-framed, and

- $R_{+}$and $R_{-}$are contained in mutually disjoint three-dimensional balls.

Proof. Let $X$ be a four-dimensional 2-handlebody such that $\partial X=M$ and the restriction of the unique spin structure on $X$ to $M$ is $s$. Let $L_{0}$ be the Kirby diagram of $X$. We denote by $n(K)$ the integer-valued framing of each component $K$ of $L_{0}$. Remark that all $n(K)$ are even since $X$ admits a spin structure. Take two unknots $K_{-}$ and $K_{+}$which are contained in mutually disjoint three-dimensional ball in $S^{3} \backslash L_{0}$. Put $n\left(K_{-}\right)=0$ and $n\left(K_{+}\right)=2$.

Fix an unknotted embedding $\psi_{0}$ of the solid torus. Recall that any link can be $\psi_{0}$-braided by Alexander's theorem (see e.g. [2], Theorem 2.1). By Proposition 3.21, there exists a total foliation $\left(\mathcal{F}_{0}^{i}\right)_{i=1}^{3}$ on $S^{3}$ such that each component $K$ of $L_{0} \cup$ $K_{-} \cup K_{+}$is contained in an $\mathcal{R}$-component with $\left(\omega\left(K ; \psi_{0}\right)+n\left(K ; \psi_{0}\right)\right)$-framing. Lemma 3.17 implies that these $\mathcal{R}$-components are odd-framed. By Lemma 3.22, we 
can modify $\left(\mathcal{F}_{0}^{i}\right)_{i=1}^{3}$ so that the framing of an $\mathcal{R}$-component $R$ is $n(C(R))-1$. Then, the standard surgery on $L_{0}$ (not $L_{0} \cup K_{-} \cup K_{+}$) produces a total foliation $\left(\mathcal{F}^{i}\right)_{i=1}^{3}$ on $M$. It is easy to see that each $K_{ \pm} \subset M$ is the core of a $( \pm 1)$-framed unknotted $\mathcal{R}$-component of $\left(\mathscr{F}^{i}\right)_{i=1}^{3}$. Proposition 2.14 implies that the spin structure given by $\left(\mathcal{F}^{i}\right)_{i=1}^{3}$ is $s$.

Remark The last sentence of Paragraph 14 of Chapter 7 (p. 71) of [10] seems incorrect. In fact, branched double covering along the unknot changes the framing of braided knots in general. For example, Figure 13 illustrates a branched double
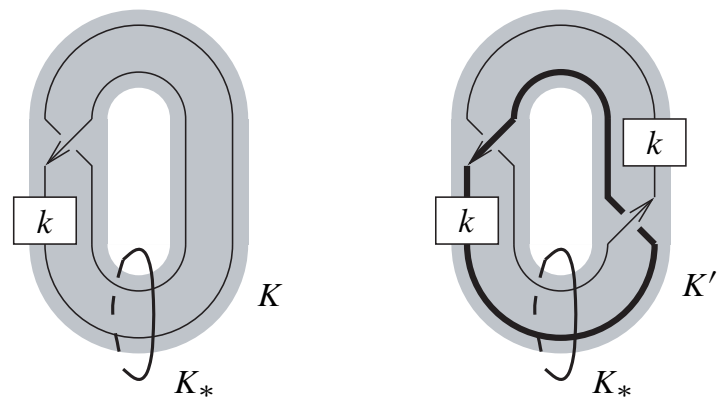

Figure 13. Double covering of a solid torus.

covering along the unknot $K_{*}$. The box represents a tangle where the difference of the numbers of positive and negative crossings is $k$. Suppose that the knot $K$ in the left-side of the figure has the blackboard framing, which is equal to the $(k+1)$ framing. In the right-side of the figure, which is a double covering of the left-side, the lift $K^{\prime}$ of the framed knot $K$ has the blackboard framing, which is equal to the $k$-framing. Hence, the knot $K$ is isotopic to $K^{\prime}$ as a knot, but is not isotopic to $K^{\prime}$ as a framed knot. It is because one positive crossing in the left-side is not counted in the right-side. The same phenomenon occurs in the setting in Chapter 7 of [10].

3.6. Proof of Theorem 1.1. First, we construct a suitable total foliation on $S^{3}$. Let $\left(\mathcal{R}_{+}^{i}\right)_{i=1}^{3}$ be the positive total Reeb foliation on $S^{3}$, that is, a total foliation consisting of two (-1)-framed unknotted $\mathcal{R}$-components.

Lemma 3.24. For any integer $n$, there exists a total foliation $\left(\mathscr{G}_{n}^{i}\right)_{i=1}^{3}$ on $S^{3}$ with unknotted $\mathcal{R}$-components $R_{+}$and $R_{-}$such that $H\left(\left(\mathcal{E}_{n}^{i}\right)_{i=1}^{3},\left(\mathcal{R}_{+}^{i}\right)_{i=1}^{3}\right)=n, R_{+}$is $(+1)$-framed, $R_{-}$is $(-1)$-framed, and $R_{+}$and $R_{-}$are contained in mutually disjoint three-dimensional balls.

Proof. First, we show the lemma for $n=-1$. By Theorem 3.23, there exists a total foliation $\left(\mathscr{F}_{0}^{i}\right)_{i=1}^{3}$ on $S^{3}$ with unknotted $\mathcal{R}$-components $R_{+}$and $R_{-}$such that 
$R_{+}$is $(+1)$-framed, $R_{-}$is $(-1)$-framed, and they are contained in mutually disjoint three-dimensional balls $B_{+}$and $B_{-}$, respectively. Since $\overline{S^{3} \backslash R_{+}}$is an unknotted solid torus, we can take an orientation reversing diffeomorphism $\varphi$ on $S^{3}$ so that $\varphi\left(\overline{S^{3} \backslash R_{+}}\right)=R_{+}$. Then, $\overline{S^{3} \backslash R_{+}}=\varphi\left(R_{+}\right)$is a $(-1)$-framed unknotted $\mathcal{R}$ component of $\left(\varphi\left(\mathcal{F}_{0}^{i}\right)\right)_{i=1}^{3}$.

Let $\left(\mathscr{E}_{-1}^{i}\right)_{i=1}^{3}$ be the total foliation obtained by gluing $\left(\mathcal{F}_{0}^{i}\right)_{i=1}^{3}$ and $\left(\varphi\left(\mathcal{F}_{0}^{i}\right)\right)_{i=1}^{3}$ along $\mathcal{R}$-components $R_{+}$and $\varphi\left(R_{+}\right)$as in Subsection 2.4. It admits unknotted $\mathcal{R}$ components $R_{-}$and $\varphi\left(R_{-}\right)$which are contained in mutually disjoint balls $B_{-}$and $\varphi\left(B_{-}\right) \subset \varphi\left(\overline{S^{3} \backslash R_{+}}\right)=R_{+}$. Then, we have

$$
\begin{aligned}
H\left(\left(\mathscr{E}_{-1}^{i}\right)_{i=1}^{3},\left(\mathcal{R}_{+}^{i}\right)_{i=1}^{3}\right) & =H\left(\left(\mathscr{G}_{-1}^{i}\right)_{i=1}^{3},\left(\mathscr{F}^{i}\right)_{i=1}^{3}\right)+H\left(\left(\mathscr{F}^{i}\right)_{i=1}^{3},\left(\mathcal{R}_{+}^{i}\right)_{i=1}^{3}\right) \\
& =H\left(\left(\varphi\left(\mathcal{F}^{i}\right)\right)_{i=1}^{3},\left(\mathcal{R}_{+}^{i}\right)_{i=1}^{3}\right)+H\left(\left(\mathscr{F}^{i}\right)_{i=1}^{3},\left(\mathcal{R}_{+}^{i}\right)_{i=1}^{3}\right) \\
& =-1,
\end{aligned}
$$

where each equality follows from the formulas (2), (3), and (4) in Subsections 2.1 and 2.3. Since $R_{-}$and $\varphi\left(R_{-}\right)$have the $(-1)$ and $(+1)$-framings respectively, the proof for $n=-1$ is completed.

Second, we show the lemma for $n \leq-1$ by induction. Suppose that there exists a total foliation $\left(\mathscr{E}_{n}^{i}\right)_{i=1}^{3}$ which satisfies the assertion of the lemma for some $n \leq-1$. Let $R_{+}^{\prime}$ and $R_{-}^{\prime}$ be $(+1)$ and $(-1)$-framed unknotted $\mathcal{R}$-components contained in mutually disjoint balls. We construct the total foliation $\left(\mathscr{E}_{n-1}^{i}\right)_{i=1}^{3}$ by gluing $\left(\mathscr{e}_{n}^{i}\right)_{i=1}^{3}$ and $\left(\mathscr{G}_{-1}^{i}\right)_{i=1}^{3}$ along $\mathcal{R}$-components $R_{+}^{\prime}$ and $R_{-}$. By the formula (4), we have $H\left(\left(\mathcal{E}_{n-1}^{i}\right)_{i=1}^{3},\left(\mathcal{R}_{+}^{i}\right)_{i=1}^{3}\right)=n-1$. It is easy to see that $\mathcal{R}$-components $R_{-}^{\prime}$ and $\varphi\left(R_{-}\right)$satisfy the assertion of the lemma.

For $n \geq 0$, put $\left(\mathscr{E}_{n}^{i}\right)_{i=1}^{3}=\left(\varphi\left(\mathscr{E}_{-n-1}^{i}\right)\right)_{i=1}^{3}$. By the formula (3), we have $H\left(\left(\mathscr{E}_{n}^{i}\right)_{i=1}^{3},\left(\mathcal{R}_{+}^{i}\right)_{i=1}^{3}\right)=n$. It is easy to see that $\left(\mathscr{E}_{n}^{i}\right)_{i=1}^{3}$ is the required one.

Now, we show the following theorem which implies Theorem 1.1.

Theorem 3.25. Let $M$ be a closed oriented three-dimensional manifold. Any homotopy class of total plane fields on $M$ can be realized by a total foliation $\left(\mathcal{F}^{i}\right)_{i=1}^{3}$ with $(+1)$ - and (-1)-framed unknotted $\mathcal{R}$-components.

Proof. Fix a spin structure $s$ on $M$. By Theorem 3.23, we can take a total foliation $\left(\mathscr{F}^{i}\right)_{i=1}^{3}$ on $M$ such that it admits $(+1)$ and $(-1)$-framed unknotted $\mathcal{R}$-components $R_{+}$and $R_{-}$, and the spin structure given by $\left(\mathscr{F}^{i}\right)_{i=1}^{3}$ is $s$. By Proposition 2.5, it is sufficient to show that there exists a total foliation $\left(\widetilde{F}_{n}^{i}\right)_{i=1}^{3}$ on $M$ such that it admits (+1)- and (-1)-unknotted $\mathcal{R}$-components, the spin structure given by $\left(\mathcal{F}_{n}^{i}\right)_{i=1}^{3}$ is $s$, and $H\left(\left(\mathscr{F}_{n}^{i}\right)_{i=1}^{3},\left(\mathscr{F}^{i}\right)_{i=1}^{3}\right)=n$ for any given integer $n$.

Take an integer $n$. Let $\left(\mathscr{G}_{n}^{i}\right)_{i=1}^{3}$ be the total foliation on $S^{3}$ obtained in Lemma 3.24 for $n$. It admits (+1)-and (-1)-framed $\mathcal{R}$-components $R_{+}^{\prime}$ and $R_{-}^{\prime}$ which are contained in mutually disjoint balls. Let $\left(\mathcal{F}_{n}^{i}\right)_{i=1}^{3}$ be the total foliation obtained by gluing 
$\left(\mathcal{F}^{i}\right)_{i=1}^{3}$ and $\left(\mathscr{E}_{n}^{i}\right)_{i=1}^{3}$ along $\mathcal{R}$-components $R_{+}$and $R_{-}^{\prime}$. Since $R_{+}$is contained in a three-dimensional ball, $\left(\mathscr{F}_{n}^{i}\right)_{i=1}^{3}$ and $\left(\mathscr{F}^{i}\right)_{i=1}^{3}$ give the same spin structure. By Proposition 2.16, we obtain $H\left(\left(\mathcal{F}_{n}^{i}\right)_{i=1}^{3},\left(\mathscr{F}^{i}\right)_{i=1}^{3}\right)=n$. It is easy to see that $R_{-}$and $R_{+}^{\prime}$ are $(-1)$ and $(+1)$-framed unknotted $\mathcal{R}$-components of $\left(\mathcal{F}_{n}^{i}\right)_{i=1}^{3}$.

\section{Bi-contact structures}

First, we recall some basic definitions and results on contact topology. A plane field $\xi$ on a three-dimensional manifold $M$ is called a positive (resp. negative) contact structure if it is the kernel of a 1 -form $\alpha$ with $\alpha \wedge d \alpha>0$ (resp. $\alpha \wedge d \alpha<0$ ). We say a knot $K$ in $(M, \xi)$ is Legendrian if it is tangent to $\xi$. The Thurston-Bennequin invariant $\operatorname{tb}(K, \xi)$ is the integer-valued framing of a null-homologous knot $K$ given by $\xi$. The rotation $\operatorname{rot}(K, \xi)$ is the Euler number $\chi(\xi, \Sigma, K)$ of $\xi$ on a Seifert surface $\Sigma$ relative to $K$.

A contact structure $\xi$ on $M$ is called overtwisted if there exists a Legendrian unknot $K$ such that $\operatorname{tb}(K, \xi)=0$. We say $\xi$ is tight if it is not overtwisted. It is known that if $\xi$ is tight, then any null-homologous Legendrian knot $K$ satisfies the Thurston-Bennequin inequality:

$$
\operatorname{tb}(K, \xi)+\chi(\Sigma) \leq-|\operatorname{rot}(K, \xi)|,
$$

where $\chi(\Sigma)$ is the Euler number of a Seifert surface $\Sigma$ of $K$.

Theorem 4.1 (Eliashberg, [6]). Let $M$ be a three-dimensional closed manifold, any homotopy class of plane fields on $M$ contains exactly one positive (resp. negative) overtwisted contact structure up to isotopy.

The following lemma gives a criterion for the overtwistedness of a contact structure which is close to a foliation of a total foliation.

Lemma 4.2. Let $\left(\mathcal{F}^{i}\right)_{i=1}^{3}$ be a total foliation on a three-dimensional manifold $M$ and suppose it admits $a(+1)$-framed (resp. $(-1)$-framed) unknotted $\mathcal{R}$-component $R$. Then, any positive (resp. negative) contact structure which is sufficiently $C^{0}$-close to $T \mathcal{F}^{1}$ is overtwisted.

Proof. We show the assertion for positive contact structures. The proof for negative contact structures is obtained by reversing the orientation.

The foliation $\left.\mathcal{F}^{1}\right|_{\partial R}$ admits a closed leaf $C$ which is isotopic to the core of $R$ as an oriented knot in $M$. In particular, $C$ is unknotted. The foliation $\mathcal{F}^{1}$ gives the (+1)-framing on $C$.

Recall that $\partial R$ is a leaf of $\mathcal{F}^{3}$. By Lemma 2.8, if a smooth plane field $\xi$ is sufficiently $C^{0}$-close to $T \mathscr{F}^{1}$, there exists a closed curve $C_{\xi}$ in $\partial R$ which is tangent 
to $\xi \cap T \mathcal{F}^{3}$ and isotopic to $C$ in $\partial R$. The curve $C_{\xi}$ is unknotted in $M$, and hence, it bounds a disk $D_{\xi}$. Since $\xi \cap T \mathcal{F}^{3}$ gives an trivialization of $\xi$ on $D_{\xi}$, we have $\operatorname{rot}\left(C_{\xi}, \xi\right)=0$.

By the transversality, $\mathscr{F}^{1}$ and $\mathscr{F}^{3}$ define the same framing on $C$, and $\xi$ and $\mathscr{F}^{3}$ define the same framing on $C_{\xi}$. Hence, the framing on $C_{\xi}$ given by $\xi$ is $(+1)$. In particular, $\operatorname{tb}\left(C_{\xi}, \xi\right)=+1$. It violates the Thurston-Bennequin inequality since

$$
\operatorname{tb}\left(C_{\xi}, \xi\right)+\chi\left(D_{\xi}\right)=2>0=\left|\operatorname{rot}\left(C_{\xi}, \xi\right)\right| .
$$

Now, we prove Theorem 1.5. Let $M$ be a closed and oriented three-dimensional manifold. Fix a pair $(\xi, \eta)$ of positive and negative contact structures such that they are homotopic as plane fields and their Euler class is zero. Then, there exists a total plane field $\left(\xi^{i}\right)_{i=1}^{3}$ on $M$ such that $\xi^{i}$ is homotopic to $\xi$ and $\eta$ for $i=1,2,3$. By Theorem 3.25, $\left(\xi_{i}\right)_{i=1}^{3}$ is homotopic to a total foliation $\left(\mathcal{F}^{i}\right)_{i=1}^{3}$ on $M$ which admits $(+1)$ and $(-1)$-framed unknotted $\mathcal{R}$-components.

By the fundamental theorem of confoliations ([7], Theorem 2.4.1) we can take a bi-contact structure $\left(\xi_{*}, \eta_{*}\right)$ on $M$ so that $\xi_{*}$ is $C^{0}$-sufficiently close to $\mathcal{F}^{1}$ and $\eta_{*}$ is $C^{0}$-sufficiently close to $\mathcal{F}^{2}$. Lemma 4.2 implies that both $\xi_{*}$ and $\eta_{*}$ are overtwisted. By Theorem 4.1, $\xi_{*}$ and $\eta_{*}$ are isotopic to $\xi$ and $\eta$ as contact structures, respectively.

\section{References}

[1] J. S. Birman, On braid groups. Comm. Pure Appl. Math. 22 (1969), 41-72. Zbl 0157.30904 MR 0234447

[2] J. S. Birman, Braids, links, and mapping class groups. Ann. of Math. Stud. 82, Princeton University Press, Princeton, N.J., 1974. Zbl 0305.57013 MR 0375281

[3] A. Candel and L. Conlon, Foliations. II. Graduate Stud. Math. 60, Amer. Math. Soc., Providence, RI, 2003. Zbl 1035.57001 MR 1994394

[4] E. Dufraine, About homotopy classes of non-singular vector fields on the three-sphere. Qual. Theory Dyn. Syst. 3 (2002), no. 2, 361-376,. Zbl 1057.37016 MR 2020693

[5] E. Dufraine, Classes d'homotopie de champs de vecteurs Morse-Smale sans singularité sur les fibrés de Seifert. Enseign. Math. (2) 51 (2005), no. 1-2, 3-30. Zbl 1098.37026 MR 2154619

[6] Y. M. Eliashberg, Classification of overtwisted contact structures on 3-manifolds. Invent. Math. 98(1989), no. 3, 623-637. Zbl 0684.57012 MR 1022310

[7] Y. M. Eliashberg and W. P. Thurston, Confoliations. Univ. Lecture Ser. 13, Amer. Math. Soc., Providence, RI, 1998. Zbl 0893.53001 MR 1483314

[8] R. E. Gompf and A. I. Stipsicz, 4-manifolds and Kirby calculus. Grad. Stud. Math. 20, Amer. Math. Soc., Providence, RI, 1999. Zbl 0933.57020 MR 1707327

[9] J. González-Meneses, New presentations of surface braid groups. J. Knot Theory Ramifications 10 (2001), no. 3, 431-451. Zbl 1030.20024 MR 1825967 
[10] D. Hardorp, All compact orientable three dimensional manifolds admit total foliations. Mem. Amer. Math. Soc. 26 (233), 1980. Zbl 0435.57005 MR 2213034

[11] J. Milnor, Spin structures on manifolds. Enseignement Math. (2) 9 (1963), 198-203. Zbl 0116.40403 MR 0157388

[12] Y. Mitsumatsu, Anosov flows and non-Stein symplectic manifolds. Ann. Inst. Fourier (Grenoble) 45 (1995), no. 5, 1407-1421. Zbl 0834.53031 MR 1370752

[13] Y. Mitsumatsu, Foliations and contact structures on 3-manifolds. In Foliations: geometry and dynamics (Warsaw, 2000), World Scientific Publishing, River Edge, NJ, 2002, 75-125. Zbl 1008.57003 MR 1882766

[14] D. Rolfsen, Knots and links. Corrected reprint of the 1976 original, Math. Lecture Ser. 7, Publish or Perish Inc., Houston, TX, 1990. Zbl 0339.55004 MR 0515288

[15] I. Tamura and A. Sato, On transverse foliations. Inst. Hautes Études Sci. Publ. Math. 54 (1981), 205-235. Zbl 0484.57016 MR 0644557

[16] J. W. Wood, Foliations on 3-manifolds. Ann. of Math. (2) 89 (1969), 336-358. Zbl 0176.21402 MR 0248873

Received October 19, 2007; revised June 15, 2009

Masayuki Asaoka, Department of Mathematics, Kyoto University, 606-8502 Kyoto, Japan

E-mail: asaoka@math.kyoto-u.ac.jp

Emmanuel Dufraine; e-mail: emmanuel.dufraine@gmail.com.

Takeo Noda, Department of Mathematics, Toho University, 274-8510 Chiba, Japan

E-mail: noda@c.sci.toho-u.ac.jp 